\title{
Selection of most effective COVID-19 virus protector using a novel MCGDM technique under linguistic generalised spherical fuzzy environment
}

\author{
Tipu Sultan Haque ${ }^{1}$. Shariful Alam ${ }^{1}$. Avishek Chakraborty ${ }^{2}$
}

Received: 27 August 2021 / Revised: 7 December 2021 / Accepted: 6 January 2022 /

Published online: 2 March 2022

(c) The Author(s) under exclusive licence to Sociedade Brasileira de Matemática Aplicada e Computacional 2022

\begin{abstract}
In this article, we have introduced a new linguistic generalized spherical fuzzy set by combining the idea of generalized spherical fuzzy set and linguistic fuzzy set. Linguistic generalized spherical fuzzy set is described by linguistic positive, linguistic neutral and linguistic negative membership degrees with the condition that the square sum of its linguistic membership degrees is less than or equal to 3 which deal with the uncertain and imprecise information in decision making in a much more suitable way. We have discussed some basic operations of linguistic generalized spherical fuzzy sets and introduced new score and accuracy functions to compare any two linguistic generalized spherical fuzzy numbers. We have developed various types of aggregation operators based on the newly defined linguistic generalized spherical fuzzy set, which have been manifested to construct a new multi-criteria group decision-making technique. Numerical example has been presented to demonstrate the proposed model. Finally, sensitivity and comparative analysis is performed to show the reliability and efficiency of the new multi-criteria group decision-making technique.
\end{abstract}

Keywords Linguistic generalized spherical fuzzy number (LGSFN) · Power aggregation (PA) operators · Multi-criteria group decision-making (MCGDM) · COVID-19 virus protector

Mathematics Subject Classification 03E72 · 47S40

\section{Introduction}

Decision making theory has been widely studied in the branch of mathematical science, social science, economics, medical science and engineering for a long time and several theoretical

Communicated by Graçaliz Pereira Dimuro.

$凶$ Avishek Chakraborty

avishek.chakraborty@aot.edu.in; tirtha.avishek93@gmail.com

1 Department of Mathematics, Indian Institute of Engineering Science and Technology, Shibpur, Howrah 711103, India

2 Department of Engineering Science, Academy of Technology, Adisaptagram, Hooghly 712502, India 
improvements have been done and still going on with respect to different characteristics and features. It can be implemented in various fields under different conditions in a crisp environment. Though, to development of the concept of impreciseness in numerical data, fuzzy set (FS) (Zadeh 1965), intuitionistic fuzzy set (IFS) (Attanassov 1986), neutrosophic set (NS) (Smarandache 1999) theories are the most flourishing ones to portray the criterion values in terms of membership degrees. Several efforts have been completed by different researchers in handing out the information using distinct uncertain parameters and operators under these decision making areas (Uluçay et al. 2019; Karaaslan and Hayat 2018; Karaaslan 2018; Garg 2019; Chakraborty et al. 2021, 2020; Haque et al. 2021). After the invention of Pythagorean fuzzy set (Yager 2013; Yager and Abbasov 2013), researchers extended its concept into three-dimensional structures to form spherical fuzzy set (SFS) and T-spherical fuzzy set (TSFS) (Mahmood et al. 2019) and applied it in various real-life related multicriteria group decision making (MCGDM) problems. In SFS, researchers considered the sum of the square of membership degrees to be less than equal to 1 and for the case of TSFS, the n-th sum of the square membership degrees is less than equal to 1 . In this current era, spherical fuzzy sets and TSFSs concepts are widely examined by various other researchers (Smarandache 2019; Gündogdua and Kahramana 2019; Jin et al. 2019; Ullah et al. 2020; Ashraf et al. 2019; Ashraf and Abdullah 2019) explicitly and established some noble work on it. Now, if anyone takes fuzzy numbers like $<0.6,0.7,0.9\rangle,<0.8,0.9,0.9>$ then it fails to satisfy the definition of spherical fuzzy number as the sum of the square of its component is not less than or equal to 1 . To grab this kind of problem, Haque et al. (2020) extended this concept into generalised spherical fuzzy number (GSFN) after some crucial structural modification on SFN which is the particular case of single-valued spherical neutrosophic numbers (Smarandache 2017). Till now, only a few works have been done in this GSFN field (Haque et al. 2020; Güner and Aygün 2020) and still a massive open space of research is left here.

As research goes on, the researchers are very much interested to develop new MCDM/MCGDM techniques using various types of aggregation operators in different uncertain environments. To enrich the MCDM/MCDGM technique, researchers have introduced various operators like Einstein operator (Munir et al. 2020; Rahman 2019), Hamacher operator (Jin et al. 2021), Domby operator (Karaaslan and Dawood 2021) in various uncertain environments. Sinani et al. (2020) developed Dombi-Hamy mean operator and applied it to evaluate third-party logistic providers. Ali et al. (2021) introduced Einstein geometric aggregation for a new complex interval-valued Pythagorean fuzzy environment and applied it in a green supplier chain management problem. Pamučar and Janković (2020) initiated the hybrid interval rough weighted Pow-Heronian operator in the MCDM field.

In the real-life problem, there are many situations, when the decision-maker's opinion or presences cannot be expressed by a quantitative form but maybe in qualitative one, Thus, in this scenario, exact numbers are not the suitable option to express such type of qualitative information. For this reason a new concept, namely, linguistic variables (LVs) (Zadeh 1975) have been recognized to grab the information which cannot be approximated by accurate numbers. Due to the enormous significance of linguistic variables, it is now one of the burning topics of impreciseness-based decision making research. Li et al. (2017) manifested the linguistic neutrosophic sets and generated Heronian mean operators, Fang and Ye (2017) established most fundamental aggregation operators on Linguistic neutrosophic number, Garg and Nancy (2018) incorporated AOs under linguistic single valued neutrosophic environment. Apart from this, various researchers like (Ashraf et al. 2018; Liu et al. 2020; Jin et al. 2019; Garg 2018) were done lots of interesting work in this linguistic environment and MCGDM environment under the different types of uncertain numbers. 


\subsection{Motivation}

However, from the literature, we see that in MCDM/MCGDM technique, it is considered that all the criteria are independent of each other. In other words, all these aggregation operators do not consider the inter-relation between the values being fused. But in a real-life situation, we observed that the criteria are directly dependent on each other i.e each criterion gives an influence on the other criteria. To consider this effect in the decision-making technique, the concept of power aggregation (PA) (Yager 2001; Xu and Yager 2010) operators have been introduced. After the invention of PA operators, $\mathrm{Xu}$ (2011) initiated PA operators for intuitionistic fuzzy number and apply it to the MCGDM problem. Then, Wei and Lu (2018) put forward PA operators into Pythagorean fuzzy numbers. Yang and Li (2016) introduced PA operators for SVNNs. Nancy (2019) defined LSVN power aggregation operators using it to solve the MCGDM problem. Garg (2021) introduced T-spherical power aggregation operators and gave their application in the MCGDM field. Also, we know GSFN is the most generalized version of fuzzy numbers which can deal with a human opinion involving complex uncertain situations in a more appropriate and meaningful way. Furthermore, in the real field, decision-makers sometimes prefer to give their preferences in terms of the qualitative form instead of exact numbers. Thus, LVs are important and preferable tools to express the qualitative view of decision-makers. Keeping the advantages of PA operators, GSFNs and LVs in mind, we introduced linguistic generalised spherical fuzzy PA operators namely LGSFPA, LGSFPWA, LGSFPG and LGSFPWG in this article.

\subsection{Advantages of linguistic generalised spherical fuzzy PA operators}

We have already discussed the advantages of LVs, GSFNs and PA operators in the previous section. Here, in this article, we have introduced linguistic generalised spherical fuzzy PA operators just amalgamating the above three operators. The advantages of newly developed linguistic generalised spherical fuzzy PA operators are listed below:

(i) PA operator for GSFNs has been not defined in the existing studies till today. We have introduced it in our paper and several properties of GSFNs have been elaborately discussed in our paper. Therefore, our work has been enriched theoretically on GSFNs and generalised in real-life applications in several application fields.

(ii) linguistic generalised spherical fuzzy PA operators consider the interrelation of the criteria but other existing operators fail to do so.

(iii) In the case of linguistic generalised spherical fuzzy PA operators, decision-makers can take the larger range of fuzzy numbers but in the case of spherical fuzzy numbers, decision-makers cannot do so.

(iv) Linguistic generalised spherical fuzzy PA operators consider neutral membership degree together with positive and negative membership degrees while PA operators of IFN (Jiang et al. 2018) and PYFN (Wei and Lu 2018) only consider the positive and negative membership degrees. The inclusion of this neutral membership degree makes our result more appropriate.

\subsection{Novelty}

In this current work, we create various types of PA operators in the LGSF environment. The LGSFN can undoubtedly explicit the uncertain subjective data in the most ideal manner and 
PA operators give more versatility in the data aggregation process. To get the advantage of both LGSFN and PA, we introduced PA operators for LGSFNs of two kinds: The first kind of PA operators aggregates the input data by assigning the weights based on the defined support function and the second kind of power operators considers not only the existing evaluated weights but also use the weights evaluated from defined support function. The main contributions of the article are given as follows

(i) Firstly, we define LGSFNs with the help of LFS and GSFNs.

(ii) Then, we introduce a new score and accuracy function for LGSFN.

(iii) Here, we develop various types of PA operators namely LGSFPA, LGSFPWA, LGSFPG and LGSFPWG.

(iv) Then, we present an MCGDM technique for solving a numerical problem and afterward apply this approach to solve a numerical example.

(v) Finally, a rigorous sensitivity analysis and comparative study have been performed to check the reliability, advancement, superiority of the proposed technique.

\subsection{Structure of the article}

The rest of this article has been given into several sections. Basic definitions related to GSFNs and PA operators are briefly given in Sect. 2. Linguistic generalized spherical fuzzy number and its corresponding properties have been shown in Sect. 3. In Sect. 4, we defined new PA operations based on LGSFNs. In Sect. 5, an MCGDM technique has been manifested based on proposed PA operators. In Sect. 6, a numerical example has been done along with the sensitivity analysis and comparative analysis. Finally, in Sect. 7 the article is concluded.

\section{Basic preliminaries}

Definition 2.1 (Haque et al. 2020) Let $\mathrm{R}$ be a universe of discourse. Then

$$
\widetilde{G}_{S P H}=\{\langle r, \alpha(r), \beta(r), \gamma(r)\rangle ; r \in R\}
$$

is called GSFS on $\mathrm{R}$, where $\alpha: R \rightarrow[0,1], \beta: R \rightarrow[0,1], \gamma: R \rightarrow[0,1]$ and also satisfy the condition $\alpha^{2}(r)+\beta^{2}(r)+\gamma^{2}(r) \leq(\sqrt{3})^{2}$ for all $r \in R$. The numbers $\alpha(r)$, $\beta(r)$ and $\gamma(r)$ represent the positive-membership degree, neutral-membership degree and negative-membership degrees respectively of the element to the set $\widetilde{G}$. For convenience, we denote this triplet as $\widetilde{G}=\left\{\langle\alpha, \beta, \gamma\rangle\right.$, where $\left.\alpha, \beta, \gamma \in[0,1], \alpha^{2}+\beta^{2}+\gamma^{2} \leq(\sqrt{3})^{2}\right\}$ and called as a generalized spherical fuzzy number (GSFN).

Proposition 2.1 (Haque et al. 2020) Let $\widetilde{G}=\left\{\langle\alpha, \beta, \gamma\rangle, \widetilde{G_{1}}=\left\{\left\langle\alpha_{1}, \beta_{1}, \gamma_{1}\right\rangle\right.\right.$ and $\widetilde{G_{2}}=$ $\left\{\left\langle\alpha_{2}, \beta_{2}, \gamma_{2}\right\rangle\right.$ be any three GSFNs. Then, their operational rules are as follows:

(i) $(\widetilde{G})^{c}=\langle\gamma, \beta, \alpha\rangle($ complement of $\widetilde{G})$;

(ii) $\widetilde{G_{1}} \leq \widetilde{G_{2}}$ iff $\alpha_{1} \leq \alpha_{2}, \beta_{1} \geq \beta_{2}, \gamma_{1} \geq \gamma_{2}$;

(iii) $\widetilde{G_{1}}=\widetilde{G_{2}}$ iff $\widetilde{G_{1}} \leq \widetilde{G_{2}}$ and $\widetilde{G_{1}} \geq \widetilde{G_{2}}$;

(iv) $\widetilde{G_{1}} \oplus \widetilde{G_{2}}=\left\langle\sqrt{\alpha_{1}^{2}+\alpha_{2}^{2}-\alpha_{1}^{2} \alpha_{2}^{2}}, \beta_{1} \beta_{2}, \gamma_{1} \gamma_{2}\right\rangle$;

(v) $\widetilde{G_{1}} \otimes \widetilde{G_{2}}=\left\langle\alpha_{1} \alpha_{2}, \beta_{1} \beta_{2}, \sqrt{\gamma_{1}^{2}+\gamma_{2}^{2}-\gamma_{1}^{2} \gamma_{2}^{2}}\right\rangle$;

(vi) $l \widetilde{G}=\left\langle\sqrt{1-\left(1-\alpha^{2}\right)^{l}},(\beta)^{l},(\gamma)^{l}\right\rangle, l \geq 0$;

(vii) $\widetilde{G}^{l}=\left\langle\alpha^{l}, \beta^{l}, \sqrt{1-\left(1-\gamma^{2}\right)^{l}}, l \geq 0\right.$; 
(viii)

$$
l^{\widetilde{G}}= \begin{cases}\left\langle l^{\sqrt{1-\alpha^{2}}}, \sqrt{1-l^{2 \beta}}, \sqrt{1-l^{2 \gamma}}\right\rangle ; & l \in(0,1) \\ \left\langle\left(\frac{1}{l}\right)^{\sqrt{1-\alpha^{2}}}, \sqrt{1-\left(\frac{1}{l}\right)^{2 \beta}}, \sqrt{1-\left(\frac{1}{l}\right)^{2 \gamma}}\right\rangle ; & l \geq 1\end{cases}
$$

Definition 2.2 (Haque et al. 2020) Let $\widetilde{G_{i}}=\left\langle\alpha_{i}, \beta_{i}, \gamma_{i}\right\rangle(i=1,2, \ldots, k)$ be an arbitrary collection of GSFNs, then the generalized spherical weighted averaging aggregation (GSWAA) operator is given by

$$
\begin{aligned}
& \operatorname{GSWAA}\left(\widetilde{G_{1}}, \widetilde{G_{2}}, \ldots, \widetilde{G_{k}}\right) \\
& =\left\langle\left[\sqrt{\left.\left.1-\prod_{i=1}^{k}\left(1-\alpha_{i}^{2}\right)^{w_{i}}\right],\left[\prod_{i=1}^{k} \beta_{i} w_{i}\right],\left[\prod_{i=1}^{k} \gamma_{i} w_{i}\right]\right\rangle}\right.\right.
\end{aligned}
$$

where $w_{i}(i=1,2, \ldots, k)$ is the weight of $\widetilde{G_{i}}(i=1,2, \ldots, k)$ with $w_{i} \in[0,1]$ and $\sum_{i=1}^{k} w_{i}=1$.

Definition 2.3 (Güner and Aygün 2020) Let $\widetilde{G_{i}}=\left\langle\alpha_{i}, \beta_{i}, \gamma_{i}\right\rangle(i=1,2, \ldots, k)$ be an arbitrary collection of GSFNs, then the generalized spherical weighted geometric aggregation (GSWGA) operator is given by

$$
\begin{aligned}
& \operatorname{GSWGA}\left(\widetilde{G_{1}}, \widetilde{G_{2}}, \ldots, \widetilde{G_{k}}\right) \\
& =\left\langle\left[\prod_{i=1}^{k} \alpha_{i}{ }^{w_{i}}\right],\left[\prod_{i=1}^{k} \beta_{i} w_{i}\right],\left[\sqrt{1-\prod_{i=1}^{k}\left(1-\gamma_{i}^{2}\right)^{w_{i}}}\right]\right\rangle
\end{aligned}
$$

where $w_{i}(i=1,2, \ldots, k)$ is the weight of $\widetilde{G_{i}}(i=1,2, \ldots, k)$ with $w_{i} \in[0,1]$ and $\sum_{i=1}^{k} w_{i}=1$.

Definition 2.4 Let $\widetilde{L}_{1}=\left\langle l_{\alpha_{1}}, l_{\beta_{1}}, l_{\gamma_{1}}\right\rangle$ and $\widetilde{L}_{2}=\left\langle l_{\alpha_{2}}, l_{\beta_{2}}, l_{\gamma_{2}}\right\rangle$ be any two LGSFN. Then distance between $\widetilde{L}_{1}$ and $\widetilde{L}_{2}$ is defined as

$$
d\left(\widetilde{L}_{1}, \widetilde{L}_{2}\right)=\frac{\frac{\alpha_{1}-\alpha_{2}}{\alpha_{1}+\alpha_{2}}+\frac{\beta_{1}-\beta_{2}}{\beta_{1}+\beta_{2}}+\frac{\gamma_{1}-\gamma_{2}}{\gamma_{1}+\gamma_{2}}}{3}
$$

Definition 2.5 (Yager 2001) Suppose, we have a collection of ' $k^{\prime}$ values $a_{1}, a_{2}, \cdots, a_{k}$. Then power average (PA) operator is defined as follows

$$
\operatorname{PA}\left(a_{1}, a_{2}, \ldots, a_{k}\right)=\frac{\sum_{s=1}^{k}\left(1+S\left(a_{s}\right)\right) a_{s}}{\sum_{s=1}^{k}\left(1+S\left(a_{s}\right)\right)}
$$

where $S\left(a_{s}\right)=\sum_{t=1, t \neq s}^{k} \operatorname{Sup}\left(a_{s}, a_{t}\right)$ and $\operatorname{Sup}\left(a_{s}, a_{t}\right)$ is the support from $a_{s}$ to $a_{t}$ which is defined as $\operatorname{Sup}\left(a_{s}, a_{t}\right)=1-d\left(a_{s}, a_{t}\right)$ and also maintain the following properties

$$
\begin{aligned}
& \text { (i) } \operatorname{Sup}\left(a_{s}, a_{t}\right) \in[0,1] \\
& \text { (ii) } \operatorname{Sup}\left(a_{s}, a_{t}\right)=\operatorname{Sup}\left(a_{t}, a_{s}\right) \\
& \text { (iii) } \operatorname{Sup}\left(a_{s}, a_{t}\right) \geq \operatorname{Sup}\left(a_{p}, a_{q}\right) \text { if } d\left(a_{s}, a_{t}\right) \leq d\left(a_{t}, a_{s}\right)
\end{aligned}
$$


Definition 2.6 (Xu and Yager 2010) Let, $a_{1}, a_{2}, \ldots, a_{k}$ be a collection of ' $k$ ' values. Then power geometric (PG) operator is defined as follows

$$
\mathrm{PG}\left(a_{1}, a_{2}, \ldots, a_{k}\right)=\prod_{s=1}^{k}\left(a_{s}\right)^{\frac{\left(1+S\left(a_{s}\right)\right)}{\sum_{s=1}^{k}\left(1+S\left(a_{s}\right)\right)}}
$$

where $S\left(a_{s}\right)=\sum_{t=1, t \neq s}^{k} \operatorname{Sup}\left(a_{s}, a_{t}\right)$ and $\operatorname{Sup}\left(a_{s}, a_{t}\right)$ is the support from $a_{s}$ to $a_{t}$ which also maintain the three properties in the definition 2.2.

\section{Linguistic generalized spherical fuzzy set}

Definition 3.1 Let $\mathrm{R}$ be a universe set and $T=\left\{l_{\alpha}: l_{0} \leq l_{\alpha} \leq l_{m}: \alpha \in[0, m]\right\}$ be a continues linguistic term set. Then, a LGSFS is defined as follows

$$
\widetilde{L}=\left\{r, l_{\alpha}(r), l_{\beta}(r), l_{\gamma}(r): r \in R\right\}
$$

where $l_{\alpha}(r), l_{\beta}(r), l_{\gamma}(r) \in T$ are called the linguistic positive, neutral and negative membership degrees respectively of the element $\mathrm{r}$ to the set $\mathrm{R}$ and also satisfy the condition $(\alpha)^{2}+(\beta)^{2}+(\gamma)^{2} \leq 3 m^{2}$. The triplet $\left\langle l_{\alpha}(r), l_{\beta}(r), l_{\gamma}(r)\right\rangle$ is denoted by $\widetilde{L}=\left\langle l_{\alpha}, l_{\beta}, l_{\gamma}\right\rangle$ and called as linguistic generalized spherical fuzzy number (LGSFN).

Proposition 3.1 Let $\widetilde{L}_{1}=\left\langle l_{\alpha_{1}}, l_{\beta_{1}}, l_{\gamma_{1}}\right\rangle$ and $\widetilde{L}_{2}=\left\langle l_{\alpha_{2}}, l_{\beta_{2}}, l_{\gamma_{2}}\right\rangle$ be any two LGSFN, $l_{\alpha_{i}}, l_{\beta_{i}}, l_{\gamma_{i}} \in T=\left\{l_{\alpha}: l_{0} \leq l_{\alpha} \leq l_{m}: \alpha \in[0, m]\right\}(i=1,2)$

(i) $\widetilde{L}_{1}=\widetilde{L}_{2}$ iff $l_{\alpha_{1}}=l_{\alpha_{2}}, l_{\beta_{1}}=l_{\beta_{2}}, l_{\gamma_{1}}=l_{\gamma_{2}}$

(ii) $\widetilde{L}_{1} \cap \widetilde{L}_{2}=\left\langle\min \left(l_{\alpha_{1}}, l_{\alpha_{2}}\right), \max \left(l_{\beta_{1}}, l_{\beta_{2}}\right), \max \left(l_{\gamma_{2}}, l_{\gamma_{2}}\right)\right\rangle$

(iii) $\widetilde{L}_{1} \cup \widetilde{L}_{2}=\left\langle\max \left(l_{\alpha_{1}}, l_{\alpha_{2}}\right), \min \left(l_{\beta_{1}}, l_{\beta_{2}}\right), \min \left(l_{\gamma_{2}}, l_{\gamma_{2}}\right)\right\rangle$

(iv) $\widetilde{L}_{1} \leq \widetilde{L}_{2}$ iff $l_{\alpha_{1}} \leq l_{\alpha_{2}}, l_{\beta_{1}} \geq l_{\beta_{2}}, l_{\gamma_{1}} \geq l_{\gamma_{2}}$

Proposition 3.2 Let $\widetilde{L}_{1}=\left\langle l_{\alpha_{1}}, l_{\beta_{1}}, l_{\gamma_{1}}\right\rangle$ and $\widetilde{L}_{2}=\left\langle l_{\alpha_{2}}, l_{\beta_{2}}, l_{\gamma_{2}}\right\rangle$ be any two LGSFN, $l_{\alpha_{i}}, l_{\beta_{i}}, l_{\gamma_{i}} \in T=\left\{l_{\alpha}: l_{0} \leq l_{\alpha} \leq l_{m}: \alpha \in[0, m]\right\}(i=1,2)$ with $\mu>0$. Then, their operational rules are as follows

(i) $\widetilde{L}_{1} \oplus \widetilde{L}_{2}=\left\langle l_{m \sqrt{\left(\frac{\alpha_{1}}{m}\right)^{2}+\left(\frac{\alpha_{2}}{m}\right)^{2}-\left(\frac{\alpha_{1} \alpha_{2}}{m^{2}}\right)^{2}}}, l_{m\left(\frac{\beta_{1} \beta_{2}}{m^{2}}\right)}, l_{m\left(\frac{\gamma_{1} \gamma_{2}}{m^{2}}\right)}\right\rangle$

(ii) $\widetilde{L}_{1} \otimes \widetilde{L}_{2}=\left\langle l_{m\left(\frac{\alpha_{1} \alpha_{2}}{m^{2}}\right)}, l_{m \sqrt{\left(\frac{\beta_{1}}{m}\right)^{2}+\left(\frac{\beta_{2}}{m}\right)^{2}-\left(\frac{\beta_{1} \beta_{2}}{m^{2}}\right)^{2}}}, l_{m \sqrt{\left(\frac{\gamma_{1}}{m}\right)^{2}+\left(\frac{\gamma_{2}}{m}\right)^{2}-\left(\frac{\gamma_{1} \gamma_{2}}{m^{2}}\right)^{2}}}\right\rangle$

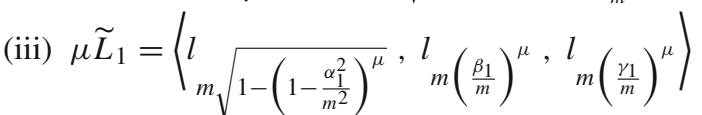

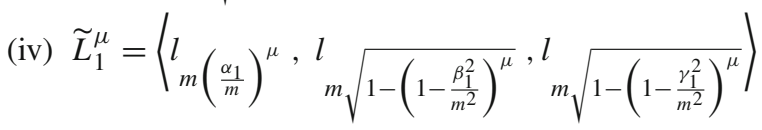

Theorem 3.1 Let $\widetilde{L}_{s}=\left\langle l_{\alpha_{s}}, l_{\beta_{s}}, l_{\gamma_{s}}\right\rangle(s=1,2)$ be any two LGSFNs, then $\widetilde{L}_{1} \oplus \widetilde{L}_{2}, \widetilde{L}_{1} \otimes \widetilde{L}_{2}$, $\mu \widetilde{L}_{1}, \widetilde{L}_{1}^{\mu}$ are also LGSFNs.

Proof Since $\widetilde{L}_{s}=\left\langle l_{\alpha_{s}}, l_{\beta_{s}}, l_{\gamma_{s}}\right\rangle(s=1,2)$ be two LGSFNs, then $\alpha_{s}, \beta_{s}, \gamma_{s} \in[0, m]$ and $(\alpha)^{2}+(\beta)^{2}+(\gamma)^{2} \leq 3 m^{2}$. Therefore, $1-\frac{\alpha_{s}^{2}}{m^{2}} \leq 1, \frac{\beta_{s}}{m} \leq 1, \frac{\gamma_{s}}{m} \leq 1$. Hence, 
$1-\left(1-\frac{\alpha_{1}^{2}}{m^{2}}\right)\left(1-\frac{\alpha_{2}^{2}}{m^{2}}\right) \leq 1$ which implies $\sqrt{1-\left(1-\frac{\alpha_{1}^{2}}{m^{2}}\right)\left(1-\frac{\alpha_{2}^{2}}{m^{2}}\right)} \leq 1$. Therefore,

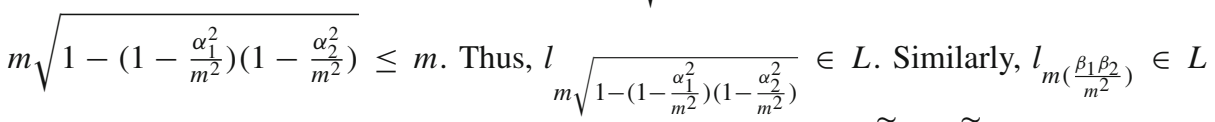
and $l_{m\left(\frac{\gamma_{1} \gamma_{2}}{m^{2}}\right)} \in L$. Then, by definition of LGSFN, we can say that $\widetilde{L}_{1} \bigoplus \widetilde{L}_{2}$ is also LGSFN. Similarly, we can proved that $\widetilde{L}_{1} \otimes \widetilde{L}_{2}, \mu \widetilde{L}_{1}, \widetilde{L}_{1}^{\mu}$ are also LGSFNs.

\subsection{Comparison of LGSFNs based on score and accuracy functions}

The score function and accuracy function are one of the important tools for determining the ranking order of LGSFNs. Here, firstly we have developed score function and accuracy function in a significant way and then gave a ranking order technique for comparing any two LGSFNs.

Definition 3.2 Let $\widetilde{L}=\left\langle l_{\alpha}, l_{\beta}, l_{\gamma}\right\rangle$ be any LGSFN, where $l_{\alpha}, l_{\beta}, l_{\gamma} \in L$. Then, score function of $\widetilde{L}$ is defined as

$$
S(\tilde{L})=\left\langle l \sqrt{\frac{2 m^{2}+\alpha^{2}-\left(\beta^{2}+\gamma^{2}\right)}{3}}\right\rangle
$$

It is easily showed that $0 \leq \frac{2 m^{2}+\alpha^{2}-\left(\beta^{2}+\gamma^{2}\right)}{3} \leq m^{2}$ which implies that $S(\widetilde{L}) \in L$

Definition 3.3 Let $\widetilde{L}=\left\langle l_{\alpha}, l_{\beta}, l_{\gamma}\right\rangle$ be any LGSFN with $l_{\alpha}, l_{\beta}, l_{\gamma} \in L$. Then, accuracy function of $\widetilde{L}$ is defined as

$$
A(\widetilde{L})=\left\langle l \sqrt{\frac{\alpha^{2}+\beta^{2}+\gamma^{2}}{3}}\right\rangle
$$

It is easily checked that $0 \leq \frac{\alpha^{2}+\beta^{2}+\gamma^{2}}{3} \leq m^{2}$ which implies that $A(\widetilde{L}) \in L$

Now, based on the above define score and accuracy functions for any two LGSFNs, we will give the following comparison rules for ranking LGSFNs.

Definition 3.4 Let $\widetilde{L}_{1}$ and $\widetilde{L}_{2}$ be any two LGSFNs. Then

(i) If $S\left(\widetilde{L}_{1}\right)>S\left(\widetilde{L}_{2}\right)$ then $L_{1}>L_{2}$ where "> means "preferred to".

(ii) If $S\left(\widetilde{L}_{1}\right)=S\left(\widetilde{L}_{2}\right)$ then

(i) If $A\left(\widetilde{L}_{1}\right)>A\left(\widetilde{L}_{2}\right)$ then $L_{1}>L_{2}$

(ii) If $A\left(\widetilde{L}_{1}\right)=A\left(\widetilde{L}_{2}\right)$ then $L_{1}=L_{2}$

Example 3.1 Let $\widetilde{L}_{1}=\left\langle l_{2}, l_{3}, l_{5}\right\rangle, \widetilde{L}_{2}=\left\langle l_{6}, l_{1}, l_{4}\right\rangle$, be two LGFNs which are obtain from $T=\left\{l_{\alpha}: l_{0} \leq l_{\alpha} \leq l_{7}, \alpha \in[0,7]\right\}$. Then utilizing the definitions 3.3, we get

$$
S\left(\widetilde{L}_{1}\right)=\left\langle l \sqrt{\frac{2 \times 7^{2}+2^{2}-\left(3^{2}+5^{2}\right)}{3}}\right\rangle=\left\langle l_{4.8}\right\rangle
$$

and

$$
S\left(\tilde{L}_{2}\right)=\left\langle l \sqrt{\frac{2 \times 7^{2}+6^{2}-\left(1^{2}+4^{2}\right)}{3}}\right\rangle=\left\langle l_{6.2}\right\rangle
$$

Since $S\left(\widetilde{L}_{2}\right) \geq S\left(\widetilde{L}_{1}\right)$, then we can obtain that $\widetilde{L}_{2} \geq \widetilde{L}_{1}$. 


\section{Power aggregation operators for LGSFN}

In this section, we have introduced some PA operators for linguistic generalized spherical fuzzy set namely, LGSFPA, LGSFPWA, LGSFPG, LGSFPWG.

\subsection{Averaging aggregation operators}

Definition 4.1 Let $\widetilde{L}_{s}=\left\langle l_{\alpha_{s}}, l_{\beta_{s}}, l_{\gamma_{s}}\right\rangle(s=1,2, \ldots, k)$ be any collection of LGSFNs. Then, a linguistic generalized spherical fuzzy power averaging (LGSFPA) aggregation operator is a mapping LGSFPA: $\prod^{k} \rightarrow \prod$, is defined by

$$
\operatorname{LGSFPA}\left(\widetilde{L}_{1}, \widetilde{L}_{2}, \ldots, \widetilde{L}_{k}\right)=\frac{\sum_{s=1}^{k}\left(1+S\left(\widetilde{L}_{s}\right)\right) \widetilde{L}_{s}}{\sum_{s=1}^{k}\left(1+S\left(\widetilde{L}_{s}\right)\right)}
$$

where $\widetilde{L}_{s} \in \prod$ and $S\left(\widetilde{L}_{s}\right)=\sum_{t=1, t \neq s}^{k} \operatorname{Sup}\left(\widetilde{L}_{s}, \widetilde{L}_{t}\right)$

Theorem 4.1 The aggregated value formed using LGSFPA operator is still a LGSFN and is given by

$$
\begin{aligned}
& \operatorname{LGSFPA}\left(\widetilde{L}_{1}, \widetilde{L}_{2}, \ldots, \widetilde{L}_{k}\right)
\end{aligned}
$$

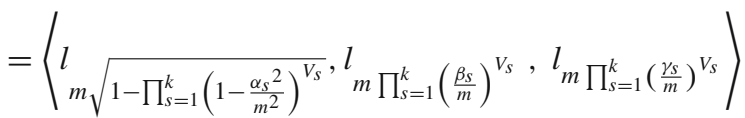

where $V_{s}=\frac{\left(1+S\left(\widetilde{L}_{s}\right)\right)}{\sum_{s=1}^{k}\left(1+S\left(\widetilde{L}_{s}\right)\right)}$, where $\widetilde{L}_{s} \in \prod$ and $S\left(\widetilde{L}_{s}\right)=\sum_{t=1, t \neq s}^{k} \operatorname{Sup}\left(\widetilde{L}_{s}, \widetilde{L}_{t}\right)$

Proof The aggregated value using the operator LGSFPA is again LGSFN which is follows from the Theorem 3.1. To prove the above result, we will use mathematical induction on $k$. For $k=2$, we have

$$
\begin{aligned}
& \operatorname{LGSFPA}\left(\widetilde{L}_{1}, \widetilde{L}_{2}\right) \\
& =\sum_{s=1}^{2} \frac{\left(1+S\left(\widetilde{L}_{s}\right)\right) \widetilde{L}_{s}}{\sum_{s=1}^{2}\left(1+S\left(\widetilde{L}_{s}\right)\right)}=V_{1} \widetilde{L}_{1}+V_{2} \widetilde{L}_{2}
\end{aligned}
$$

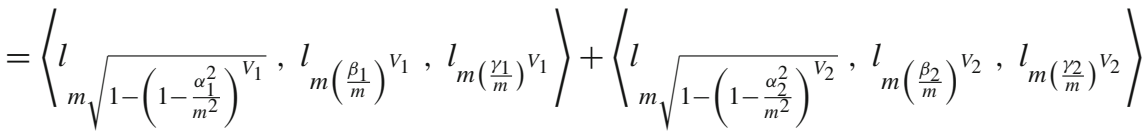

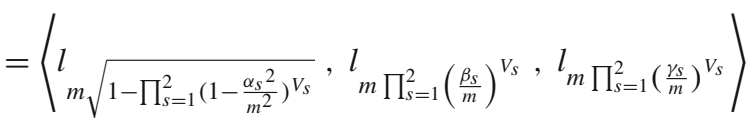

Hence, the theorem is true for $k=2$.

Let us assume that the theorem is true for $k=p$. Then

$$
\begin{aligned}
& \operatorname{LGSFPA}\left(\widetilde{L}_{1}, \widetilde{L}_{2}, \ldots, \widetilde{L}_{p}\right) \\
& =\left\langle l_{m}^{l} \sqrt{1-\prod_{s=1}^{p}\left(1-\frac{\alpha_{s}{ }^{2}}{m^{2}}\right)^{V_{s}}}, l \prod_{s=1}^{p}\left(\frac{\beta_{s}}{m}\right)^{V_{s}}, l \prod_{s=1}^{p}\left(\frac{\gamma_{s}}{m}\right)^{V_{s}}\right\rangle
\end{aligned}
$$




\section{Now,}

$$
\begin{aligned}
& \operatorname{LGSFPA}\left(\tilde{L}_{1}, \widetilde{L}_{2}, \cdots, \widetilde{L}_{p}, \tilde{L}_{p+1}\right) \\
& =V_{1} \widetilde{L}_{1}+V_{2} \widetilde{L}_{2}+\cdots+V_{p} \widetilde{L}_{p}+V_{p+1} \widetilde{L}_{p+1} \\
& =\operatorname{LGSFPA}\left(\widetilde{L}_{1}, \widetilde{L}_{2}, \cdots, \widetilde{L}_{p}\right)+V_{p+1} \widetilde{L}_{p+1} \\
& =\left\langle l \sqrt[m]{1-\prod_{s=1}^{p}\left(1-\frac{\alpha_{s}^{2}}{m^{2}}\right)^{V_{s}}}, l m \prod_{s=1}^{p}\left(\frac{\beta_{s}}{m}\right)^{V_{s}, l} m \prod_{s=1}^{p}\left(\frac{\gamma_{s}}{m}\right)^{V_{s}}\right\rangle \\
& \bigoplus\left\langle l \sqrt[l]{l \sqrt{1-\left(1-\frac{\alpha_{p+1}^{2}}{m^{2}}\right)^{V_{p+1}}}, l} m\left(\frac{\beta_{p+1}}{m}\right)^{V_{p+1}, l} m\left(\frac{\gamma_{p+1}}{m}\right)^{V_{p+1}}\right\rangle \\
& =l_{m} \sqrt{\left(1-\prod_{s=1}^{p}\left(1-\frac{\alpha_{s}^{2}}{m^{2}}\right)^{V_{s}}\right)+\left(1-\left(1-\frac{\alpha_{p+1}^{2}}{m^{2}}\right)^{V_{p+1}}\right)-\left(1-\prod_{s=1}^{p}\left(1-\frac{\alpha_{s}^{2}}{m^{2}}\right)^{V_{s}}\right)\left(1-\left(1-\frac{\alpha_{p+1}^{2}}{m^{2}}\right)^{V_{p+1}}\right)}, \\
& \left.m \prod_{s=1}^{p+1}\left(\frac{\beta_{s}}{m}\right)^{V_{s}, l} m \prod_{s=1}^{p+1}\left(\frac{\gamma_{s}}{m}\right)^{V_{s}}\right\rangle \\
& =\left\langle l \sqrt{m \sqrt{1-\prod_{s=1}^{p+1}\left(1-\frac{\alpha_{s}^{2}}{m^{2}}\right)^{V_{s}}}, l} m \prod_{s=1}^{p+1}\left(\frac{\beta_{s}}{m}\right)^{V_{s}, l} m \prod_{s=1}^{p+1}\left(\frac{\gamma_{s}}{m}\right)^{V_{s}}\right\rangle
\end{aligned}
$$

Thus, the theorem holds for $k=p+1$. Hence, by the principal of mathematical induction, the theorem holds for all positive integer $k$.

\section{Properties of the LGSFPA operator}

Let $\widetilde{L}_{s}=\left\langle l_{\alpha_{s}}, l_{\beta_{s}}, l_{\gamma_{s}}\right\rangle(s=1,2, \ldots, k)$ be any collection of LGSFNs and $\omega=$ $\left(\omega_{1}, \omega_{2}, \ldots, \omega_{k}\right)^{T}$ be the weight vector of $L_{s}(s=1,2, \ldots, k)$ with the condition $\sum_{s=1}^{k} \omega_{s}=$ $1, \omega_{s}>0$. Then LGSFPA has the following properties:

(1) (Idempotency property) If $\widetilde{L}_{s}=\widetilde{L}=\left\langle l_{\alpha}, l_{\beta}, l_{\gamma}\right\rangle$ for all $s$,

$$
\operatorname{LGSFPA}\left(\widetilde{L}_{1}, \widetilde{L}_{2}, \cdots, \widetilde{L}_{k}\right)=L
$$

Proof Since $\widetilde{L}_{s}=\widetilde{L}=\left\langle l_{\alpha}, l_{\beta}, l_{\gamma}\right\rangle$, then $\alpha_{s}=\alpha, \beta_{s}=\beta, \gamma_{s}=\gamma$ for all $s$.

Now,

$$
\begin{aligned}
& \operatorname{LGSFPA}\left(\widetilde{L}_{1}, \tilde{L}_{2}, \cdots, \widetilde{L}_{k}\right)
\end{aligned}
$$

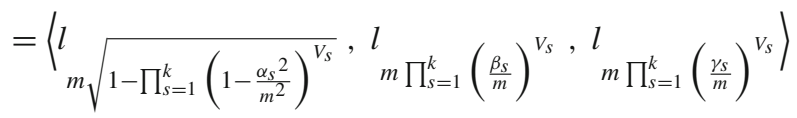

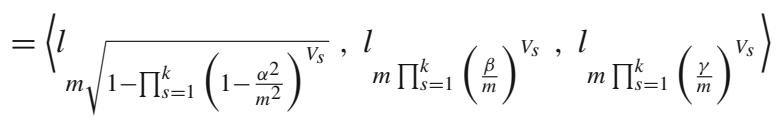

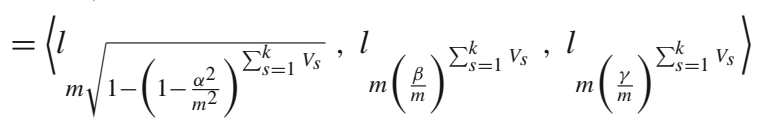

$$
\begin{aligned}
& =\left\langle l_{m \sqrt{1-\left(1-\frac{\alpha^{2}}{m^{2}}\right)}}, l_{m\left(\frac{\beta}{m}\right)}, l_{m\left(\frac{\gamma}{m}\right)}\right\rangle
\end{aligned}
$$




$$
\begin{aligned}
=\left\langle l_{\alpha}, l_{\beta}, l_{\gamma}\right\rangle \\
=\widetilde{L}
\end{aligned}
$$

(2) (Monotonicity property) Let $\widetilde{L}_{s}^{\prime}=\left\langle l_{\alpha_{s}^{\prime}}, l_{\beta_{s}^{\prime}}, l_{\gamma_{s}^{\prime}}\right\rangle(s=1,2, \ldots, k)$ be another collection of LGSFNs with the condition $l_{\alpha_{s}^{\prime}} \geq l_{\alpha_{s}}, l_{\beta_{s}^{\prime}} \leq l_{\beta_{s}}, l_{\gamma_{s}^{\prime}} \leq l_{\gamma_{s}}$. Then

$$
\operatorname{LGSFPA}\left(\widetilde{L}_{1}, \widetilde{L}_{2}, \ldots, \widetilde{L}_{k}\right) \leq \operatorname{LGSFPA}\left(\widetilde{L}_{1}^{\prime}, \widetilde{L}_{2}^{\prime}, \ldots, \widetilde{L}_{k}^{\prime}\right)
$$

Proof Since, $\quad l_{\alpha_{s}^{\prime}} \geq l_{\alpha_{s}}$

$$
\begin{aligned}
& \Longrightarrow \alpha_{s}^{\prime} \geq \alpha_{s} \\
& \Longrightarrow \frac{\left(\alpha_{s}^{\prime 2}\right)}{m^{2}} \geq \frac{\left(\alpha_{s}^{2}\right)}{m^{2}} \\
& \Longrightarrow 1-\frac{\left(\alpha_{s}^{\prime 2}\right)}{m^{2}} \leq 1-\frac{\left(\alpha_{s}^{2}\right)}{m^{2}} \\
& \Longrightarrow \prod_{s=1}^{k}\left(1-\frac{\left(\alpha_{s}^{\prime 2}\right)}{m^{2}}\right) \leq \prod_{s=1}^{k}\left(1-\frac{\left(\alpha_{s}^{2}\right)}{m^{2}}\right) \\
& \Longrightarrow 1-\prod_{s=1}^{k}\left(1-\frac{\left(\alpha_{s}^{\prime 2}\right)}{m^{2}}\right) \geq 1-\prod_{s=1}^{k}\left(1-\frac{\left(\alpha_{s}^{2}\right)}{\left.m^{2}\right)}\right. \\
& \Longrightarrow \\
& \quad \sqrt{1-\prod_{s=1}^{k}\left(1-\frac{\left(\alpha_{s}^{\prime 2}\right)}{m^{2}}\right)} \geq m \sqrt{1-\prod_{s=1}^{k}\left(1-\frac{\left(\alpha_{s}^{2}\right)}{m^{2}}\right)}
\end{aligned}
$$

Again, since $l_{\beta_{s}^{\prime}} \leq l_{\beta_{s}}$ and $l_{\gamma_{s}^{\prime}} \leq l_{\gamma_{s}}$, we can obtain that, $m \prod_{s=1}^{k}\left(\frac{\beta_{s}^{\prime}}{m}\right) \leq \prod_{s=1}^{k}\left(\frac{\beta_{s}}{m}\right)$ and $m \prod_{s=1}^{k}\left(\frac{\gamma_{s}^{\prime}}{m}\right) \leq \prod_{s=1}^{k}\left(\frac{\gamma_{s}}{m}\right)$

$$
\begin{aligned}
& \therefore \operatorname{LGSFPA}\left(\tilde{L}_{1}, \tilde{L}_{2}, \ldots, \widetilde{L}_{k}\right)
\end{aligned}
$$

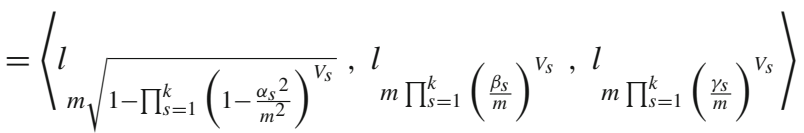

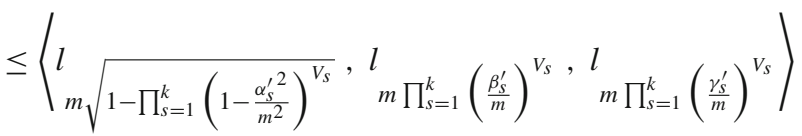

$$
\begin{aligned}
& =\operatorname{LGSFPA}\left(\widetilde{L}_{1}^{\prime}, \widetilde{L}_{2}^{\prime}, \ldots, \widetilde{L}_{k}^{\prime}\right)
\end{aligned}
$$

(3) (Boundedness property) If $\widetilde{L}_{s}^{-}=\left\langle\min _{s}\left(l_{\alpha_{s}}\right), \max _{s}\left(l_{\beta_{s}}\right), \max _{s}\left(l_{\gamma_{s}}\right)\right\rangle$ and $\widetilde{L}_{s}^{+}=$ $\left\langle\max _{s}\left(l_{\alpha_{s}}\right), \min _{s}\left(l_{\beta_{s}}\right), \min _{s}\left(l_{\gamma_{s}}\right)\right\rangle$ be LGSFNs, then

$$
L^{-} \leq \operatorname{LGSFPA}\left(\widetilde{L}_{1}, \widetilde{L}_{2}, \ldots, \widetilde{L}_{k}\right) \leq L^{+}
$$

Proof We have, $\min _{s}\left(l_{\alpha_{s}}\right) \leq l_{\alpha_{s}} \leq \max _{s}\left(l_{\alpha_{s}}\right), \max _{s}\left(l_{\beta_{s}}\right) \geq l_{\beta_{s}} \geq \min _{s}\left(l_{\beta_{s}}\right), \max _{s}\left(l_{\gamma_{s}}\right) \geq$ $l_{\gamma_{s}} \geq \min _{s}\left(l_{\gamma_{s}}\right)$, then by utilizing the monotonicity and idempotency properties, we can show that

$$
L^{-} \leq \operatorname{LGSFPA}\left(\widetilde{L}_{1}, \widetilde{L}_{2}, \ldots, \widetilde{L}_{k}\right) \leq L^{+}
$$

Definition 4.2 Let $\widetilde{L}_{s}=\left\langle l_{\alpha_{s}}, l_{\beta_{s}}, l_{\gamma_{s}}\right\rangle(s=1,2, \ldots, k)$ be any collection of LGSFNs. Then, a LGSF power weighted average (LGSFPWA) aggregation operator is a mapping LGSFPWA : $\prod^{k} \rightarrow \prod$, is defined by

$$
\operatorname{LGSFPWA}\left(\widetilde{L}_{1}, \widetilde{L}_{2}, \ldots, \widetilde{L}_{k}\right)=\bigoplus_{s=1}^{k} \Psi_{s} \widetilde{L}_{s}
$$


where, $\Psi_{s}=\frac{\phi_{s}\left(1+S\left(\widetilde{L}_{s}\right)\right)}{\sum_{s=1}^{k} \phi_{s}\left(1+S\left(\widetilde{L}_{s}\right)\right)}, S\left(\widetilde{L}_{s}\right)=\sum_{t=1, t \neq s}^{k} \operatorname{Sup}\left(\widetilde{L}_{s}, \widetilde{L}_{t}\right), \widetilde{L}_{s} \in \prod$ and $\psi_{s}$ is the weight of $\widetilde{L}_{s}$ with $\psi_{s} \geq 0$ and $\sum_{s=1}^{k} \psi_{s}=1$

Theorem 4.2 The aggregated value formed using LGSFPWA operator is still a LGSFN and is given

$$
\begin{aligned}
& \operatorname{LGSFPWA}\left(\widetilde{L}_{1}, \widetilde{L}_{2}, \ldots, \widetilde{L}_{k}\right) \\
& =\left\langle l_{m \sqrt{1-\prod_{s=1}^{k}\left(1-\frac{\alpha_{s}^{2}}{m^{2}}\right)^{\Psi_{s}}}, l}^{l} \prod_{m}^{k}\left(\frac{\beta_{s}}{m}\right)^{\Psi_{s}}, l \prod_{s=1}^{k}\left(\frac{\gamma_{s}}{m}\right)^{\Psi_{s}}\right\rangle
\end{aligned}
$$

where $\Psi_{s}=\frac{\phi_{s}\left(1+S\left(\widetilde{L}_{s}\right)\right)}{\sum_{s=1}^{k} \phi_{s}\left(1+S\left(\widetilde{L}_{s}\right)\right)}$ and $S\left(\widetilde{L}_{s}\right)=\sum_{t=1, t \neq s}^{k} \operatorname{Sup}\left(\widetilde{L}_{s}, \widetilde{L}_{t}\right), \widetilde{L}_{s} \in \prod$

Proof The proof is similar to the proof of the Theorem 4.1.

\subsection{Geometric aggregation operators}

Definition 4.3 A linguistic generalized spherical fuzzy power geometric (LGSFPG) aggregation operator is a mapping LGSFPG : $\prod^{k} \rightarrow \prod$, is defined as

$$
\operatorname{LGSFPG}\left(\widetilde{L}_{1}, \widetilde{L}_{2}, \ldots, \widetilde{L}_{k}\right)=\prod_{s=1}^{k} \widetilde{L}_{s=1}^{k}\left(1+S\left(\widetilde{L}_{s}\right)\right)
$$

where $\widetilde{L}_{s} \in \prod$ and $S\left(\widetilde{L}_{s}\right)=\sum_{t=1, t \neq s}^{k} \operatorname{Sup}\left(\widetilde{L}_{s}, \widetilde{L}_{t}\right)$

Theorem 4.3 The aggregated value using LGSFPG operator is still a LGSFN and is given by

$$
\begin{aligned}
& \operatorname{LGSFPG}\left(\widetilde{L}_{1}, \widetilde{L}_{2}, \ldots, \widetilde{L}_{k}\right) \\
& =\left\langle l{ }_{m}^{l} \prod_{s=1}^{k}\left(\frac{\alpha_{s}}{m}\right)^{V_{s}}, l \sqrt[m]{1-\prod_{s=1}^{k}\left(1-\frac{\beta_{s}^{2}}{m^{2}}\right)^{V_{s}}}, l \sqrt[m]{1-\prod_{s=1}^{k}\left(1-\frac{\gamma_{s}^{2}}{m^{2}}\right)^{V_{s}}}\right\rangle
\end{aligned}
$$

where $V_{s}=\frac{\left(1+S\left(\widetilde{L}_{s}\right)\right)}{\sum_{s=1}^{k}\left(1+S\left(\widetilde{L}_{s}\right)\right)}, \widetilde{L}_{s} \in \prod$ and $S\left(\widetilde{L}_{s}\right)=\sum_{t=1, t \neq s}^{k} \operatorname{Sup}\left(\widetilde{L}_{s}, \widetilde{L}_{t}\right)$

Proof The proof is similar to the proof of the Theorem 4.1.

Definition 4.4 A linguistic generalized spherical fuzzy (LGSF) power weighted geometric (LGSFPWG) aggregation operator is a mapping LGSFPWG : $\prod^{k} \rightarrow \prod$, is defined as

$$
\operatorname{LGSFPWG}\left(\widetilde{L}_{1}, \widetilde{L}_{2}, \ldots, \widetilde{L}_{k}\right)=\bigotimes_{s=1}^{k} \widetilde{L}_{s}^{\Psi_{s}}
$$

where $\Psi_{s}=\frac{\phi_{s}\left(1+S\left(\widetilde{L}_{s}\right)\right)}{\sum_{s=1}^{k} \phi_{s}\left(1+S\left(\widetilde{L}_{s}\right)\right)}$ and $S\left(\widetilde{L}_{s}\right)=\sum_{t=1, t \neq s}^{k} \operatorname{Sup}\left(\widetilde{L}_{s}, \widetilde{L}_{t}\right), \widetilde{L}_{s} \in \prod$ 
Theorem 4.4 The aggregated value using LGSFPWG operator is still a LGSFN and is given by

$$
\begin{aligned}
& \operatorname{LGSFPWG}\left(\widetilde{L}_{1}, \widetilde{L}_{2}, \ldots, \widetilde{L}_{k}\right)
\end{aligned}
$$

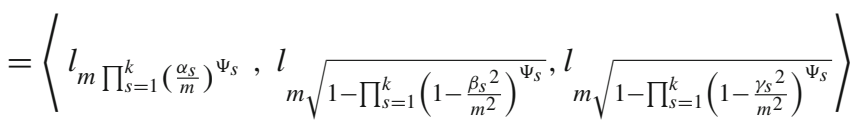

where $\Psi_{s}=\frac{\phi_{s}\left(1+S\left(\widetilde{L}_{s}\right)\right)}{\sum_{s=1}^{k} \phi_{s}\left(1+S\left(\widetilde{L}_{s}\right)\right)}$ and $S\left(\widetilde{L}_{s}\right)=\sum_{t=1, t \neq s}^{k} \operatorname{Sup}\left(\widetilde{L}_{s}, \widetilde{L}_{t}\right), \widetilde{L}_{s} \in \prod$

Proof The proof is similar to the proof of the Theorem 4.1.

\section{MCGDM technique based on power aggregation operators}

Here, we have considered a decision making problem which have' $s^{\prime}$ alternatives $M_{1}, M_{2}, \ldots, M_{s}$ and ' $t$ ' criteria $N_{1}, N_{2}, \ldots, N_{t}$ with the weight vector $\Theta=\left(\theta_{1}, \theta_{2}, \ldots, \theta_{t}\right)$ such that each $\theta_{i} \geq 0$ and also satisfy the condition $\sum_{k=1}^{t} \theta_{k}=1$. We also assume that there are ' $r$ ' set of decision-makers $D_{1}, D_{2}, \ldots, D_{r}$ which have evaluated these alternatives over the criteria and give their preferences in the form LGSFNs. Let $\Phi=\left(\phi_{1}, \phi_{2}, \ldots, \phi_{r}\right)$ be the weight vector of the decision-makers with the condition $\sum_{k=1}^{r} \phi_{k}=1$ and $\phi_{k} \geq 0$. The overall preferences values of the alternatives are characterized in the form of decision matrix $D M^{k}=\left(\widetilde{L}_{i j}^{k}\right)_{s \times t}$, where $\widetilde{L}_{i j}^{k}=\left\langle l_{\alpha_{i j}}^{k}, l_{\beta_{i} j}^{k}, l_{\gamma_{i} j}^{k}\right\rangle(i=1,2, \ldots, s)(j=1,2, \ldots, t)(k=1,2, \ldots, r)$. Depend of the above information, we proposed the following steps to select the best alternatives as follows:

Step 1: First, we construct the linguistic generalized spherical fuzzy decision matrices $D M^{k}(k=1,2, \ldots, p)$ as follows :

$$
\mathbf{D M}^{\mathbf{k}}=\begin{gathered}
M_{1} \\
M_{2} \\
\vdots \\
M_{s}
\end{gathered}\left(\begin{array}{cccc}
N_{1} & N_{2} & \cdots & N_{t} \\
\widetilde{L}_{11}^{k} & \widetilde{L}_{12}^{k} & \cdots & \widetilde{L}_{1 t}^{k} \\
\widetilde{L}_{21}^{k} & \widetilde{L}_{22}^{k} & \cdots & \widetilde{L}_{2 t}^{k} \\
\vdots & & \vdots & \vdots \\
\widetilde{L}_{s 1}^{k} & \widetilde{L}_{t 2}^{k} & \cdots & \widetilde{L}_{s t}^{k}
\end{array}\right)
$$

Step 2: Then, applying the PA operators on every individual decision matrices $D M^{k}(k=$ $1,2, \ldots, r)$ and we have overall evaluation values for the alternatives corresponding to the each decision-makers in the form of column matrices $C_{s \times 1}^{k}$ as follows

$$
C_{s \times 1}^{k}=\begin{gathered}
M_{1} \\
M_{2} \\
\vdots \\
M_{s}
\end{gathered}\left(\begin{array}{c}
\widetilde{L}_{11}^{k} \\
\widetilde{L}_{21}^{k} \\
\vdots \\
\widetilde{L}_{s 1}^{k}
\end{array}\right)
$$

Step 3: Here, we use decision-maker's weight according to the relation $\sum_{k=1}^{r} \phi_{k} C_{\mathcal{J}^{\prime} 1}^{k}$ (scalar multiplication and addition of LGSFNs) and we obtain overall preferences values $\widetilde{L}_{i 1}$ 
Fig. 1 Flowchart of the proposed MCGDM technique

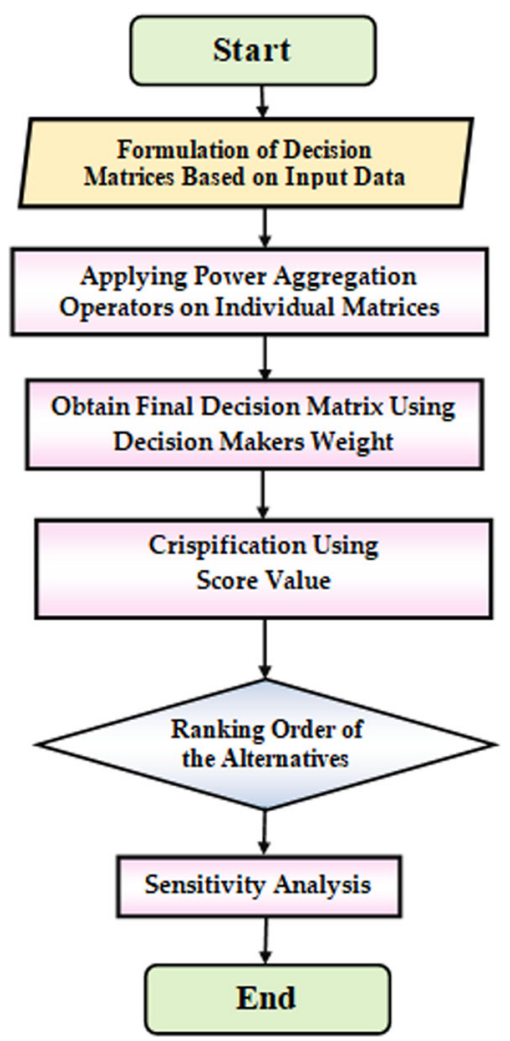

for the alternatives $M_{i}$ as follows

$$
\left.\mathbf{D M}=\begin{array}{cc}
M_{1} & \widetilde{L}_{11} \\
M_{2} & \widetilde{L}_{21} \\
\vdots & \vdots \\
M_{S} & \widetilde{L}_{s 1}
\end{array}\right)
$$

Step 4: Then, utilizing the score function according to the Definition 3.2, we get $S\left(\widetilde{L}_{j 1}\right)$ of the alternatives $M_{j}(j=1,2, \ldots, s)$.

Step 5: In this step, the alternatives are ranked and choose the best option according to the Definition 3.4.

Step 6: End.

The different steps of the above proposed MCGDM technique are shown in the Fig. 1 as follows:

\section{Numerical example}

In this current situation, people of the whole world are tensed and panicked due to the spread of COVID-19 worldwide. Several people are already died due to this harmful virus and scientists are very busy creating the vaccine of COVID-19. In this situation, people are always 
Fig. 2 Different types of COVID-19 protective equipments (alternatives)

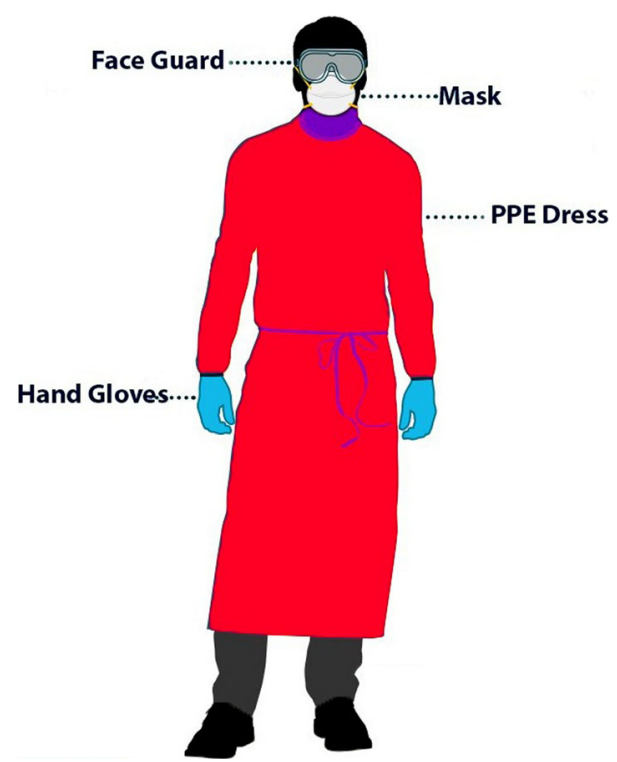

trying to protect themselves from this virus using several protectors like face guards, masks, PPE kits, gloves, etc. Also, they are focused on several parameters like efficiency, costing, reliability, reusability, etc of the protective materials. They took various opinions on it from different experts like doctors, virologists, biologists, etc and focused on the statistical data. But, the scientists are also confused and they are in dilemma to give all the answers properly. So, this becomes an important and burning decision making problem in the uncertainty domain. To resolve it burning issue, we consider an MCGDM problem in generalized spherical environment linked with four distinct protective items (Face Guard, PPE kit, Mask and Gloves) (Fig. 2) of the COVID-19 virus and their effectiveness due to the presence of four attribute functions namely efficiency, accessibility, reusability and costing. We would like to compute the most effective protective material after examining different types of scientist's opinions collected from different research sources on COVID-19 protectors. Here, we classified four dissimilar categories of scientists according to their subject division and applied suitable weights based on their research experience. Thus, it becomes an MCGDM problem having four alternatives, four attributes and three types of decision-maker. Here, we construct a MCGDM problem based on environment as follows: $M_{1}=$ Protector $1=$ Face Guard, $M_{2}$ = Protector $2=$ Mask, $M_{3}=$ Protector $3=$ PPE Dress, $M_{4}=$ Protector $4=$ Hand Gloves are the alternatives respectively. Also, $N_{1}=$ Efficiency, $N_{2}=$ Reusability, $N_{3}=$ Accessibility, $N_{4}$ $=$ Costing are the attributes whose weight vector is $(0.27,0.24,0.25,0.24)$. Let us consider there are three environment experts as decision-makers $D_{1}=$ Doctors, $D_{3}=$ Virologists, $D_{3}=$ Biologists and $D_{4}=$ Statistician having weight vector $\rho=(0.25,0.26,0.26,0.23)$. The four decision-makers $D_{1}, D_{2}, D_{3}$ and $D_{4}$ are invited to give their preferences for each alternative on each attribute with the linguistic term set $S=l_{0}=$ Extremely low(EL), $l_{1}=$ Very low(VL), $l_{2}=$ Quasi low $(\mathrm{QL}), l_{3}=\operatorname{Low}(\mathrm{L}), l_{4}=$ Semi low $(\mathrm{SL}), l_{5}=$ Standard $(\mathrm{SD}), l_{6}=$ Semi high $(\mathrm{SM}), l_{7}=\operatorname{High}(\mathrm{H}), l_{8}=$ Quasi high $(\mathrm{QH}), l_{9}=\operatorname{Very} \operatorname{high}(\mathrm{VH}), l_{10}=\operatorname{Extreme} \operatorname{high}(\mathrm{EH})$. Now, we will use our proposed MCGDM technique as follows:

Step 1: The evaluation values of alternatives over the criteria under various decision-maker in terms of decision matrices are given as follows: 


\begin{tabular}{|c|c|c|c|c|}
\hline & $N_{1}$ & $N_{2}$ & $N_{3}$ & $N_{4}$ \\
\hline \multicolumn{5}{|c|}{$D M^{1}$ : Evaluation of the alternatives under decision-maker $D_{1}$} \\
\hline$M_{1}$ & $\left\langle l_{6}, l_{8}, l_{9}\right\rangle$ & $\left\langle l_{2}, l_{6}, l_{8}\right\rangle$ & $\left\langle l_{1}, l_{7}, l_{8}\right\rangle$ & $\left\langle l_{3}, l_{6}, l_{9}\right\rangle$ \\
\hline$M_{2}$ & $\left\langle l_{8}, l_{6}, l_{8}\right\rangle$ & $\left\langle l_{7}, l_{5}, l_{7}\right\rangle$ & $\left\langle l_{8}, l_{5}, l_{8}\right\rangle$ & $\left\langle l_{5}, l_{7}, l_{9}\right\rangle$ \\
\hline$M_{3}$ & $\left\langle l_{9}, l_{7}, l_{5}\right\rangle$ & $\left\langle l_{3}, l_{7}, l_{9}\right\rangle$ & $\left\langle l_{2}, l_{5}, l_{9}\right\rangle$ & $\left\langle l_{7}, l_{6}, l_{8}\right\rangle$ \\
\hline \multirow[t]{2}{*}{$M_{4}$} & $\left\langle l_{8}, l_{5}, l_{7}\right\rangle$ & $\left\langle l_{5}, l_{6}, l_{7}\right\rangle$ & $\left\langle l_{9}, l_{2}, l_{7}\right\rangle$ & $\left\langle l_{5}, l_{8}, l_{2}\right\rangle$ \\
\hline & $N_{1}$ & $N_{2}$ & $N_{3}$ & $N_{4}$ \\
\hline \multicolumn{5}{|c|}{$D M^{2}$ : Evaluation of the alternatives under decision-maker $D_{2}$} \\
\hline$M_{1}$ & $\left\langle l_{5}, l_{1}, l_{2}\right\rangle$ & $\left\langle l_{5}, l_{7}, l_{8}\right\rangle$ & $\left\langle l_{5}, l_{4}, l_{2}\right\rangle$ & $\left\langle l_{3}, l_{5}, l_{7}\right\rangle$ \\
\hline$M_{2}$ & $\left\langle l_{3}, l_{7}, l_{8}\right\rangle$ & $\left\langle l_{6}, l_{3}, l_{7}\right\rangle$ & $\left\langle l_{3}, l_{4}, l_{7}\right\rangle$ & $\left\langle l_{8}, l_{5}, l_{8}\right\rangle$ \\
\hline$M_{3}$ & $\left\langle l_{2}, l_{8}, l_{7}\right\rangle$ & $\left\langle l_{5}, l_{7}, l_{6}\right\rangle$ & $\left\langle l_{7}, l_{3}, l_{4}\right\rangle$ & $\left\langle l_{4}, l_{5}, l_{7}\right\rangle$ \\
\hline \multirow[t]{2}{*}{$M_{4}$} & $\left\langle l_{5}, l_{6}, l_{5}\right\rangle$ & $\left\langle l_{7}, l_{6}, l_{7}\right\rangle$ & $\left\langle l_{7}, l_{1}, l_{2}\right\rangle$ & $\left\langle l_{1}, l_{2}, l_{8}\right\rangle$ \\
\hline & $N_{1}$ & $N_{2}$ & $N_{3}$ & $N_{4}$ \\
\hline \multicolumn{5}{|c|}{$D M^{3}$ : Evaluation of the alternatives under decision-maker $D_{3}$} \\
\hline$M_{1}$ & $\left\langle l_{1}, l_{4}, l_{3}\right\rangle$ & $\left\langle l_{3}, l_{4}, l_{5}\right\rangle$ & $\left\langle l_{5}, l_{2}, l_{7}\right\rangle$ & $\left\langle l_{6}, l_{5}, l_{6}\right\rangle$ \\
\hline$M_{2}$ & $\left\langle l_{2}, l_{3}, l_{5}\right\rangle$ & $\left\langle l_{7}, l_{6}, l_{5}\right\rangle$ & $\left\langle l_{9}, l_{1}, l_{2}\right\rangle$ & $\left\langle l_{8}, l_{7}, l_{8}\right\rangle$ \\
\hline$M_{3}$ & $\left\langle l_{4}, l_{5}, l_{6}\right\rangle$ & $\left\langle l_{6}, l_{7}, l_{6}\right\rangle$ & $\left\langle l_{3}, l_{4}, l_{7}\right\rangle$ & $\left\langle l_{8}, l_{2}, l_{7}\right\rangle$ \\
\hline \multirow[t]{2}{*}{$M_{4}$} & $\left\langle l_{5}, l_{4}, l_{7}\right\rangle$ & $\left\langle l_{7}, l_{8}, l_{9}\right\rangle$ & $\left\langle l_{6}, l_{4}, l_{7}\right\rangle$ & $\left\langle l_{8}, l_{5}, l_{6}\right\rangle$ \\
\hline & $N_{1}$ & $N_{2}$ & $N_{3}$ & $N_{4}$ \\
\hline \multicolumn{5}{|c|}{$D M^{4}$ : Evaluation of the alternatives under decision-maker $D_{4}$} \\
\hline$M_{1}$ & $\left\langle l_{7}, l_{5}, l_{7}\right\rangle$ & $\left\langle l_{5}, l_{6}, l_{4}\right\rangle$ & $\left\langle l_{6}, l_{5}, l_{6}\right\rangle$ & $\left\langle l_{7}, l_{6}, l_{8}\right\rangle$ \\
\hline$M_{2}$ & $\left\langle l_{2}, l_{5}, l_{6}\right\rangle$ & $\left\langle l_{2}, l_{3}, l_{8}\right\rangle$ & $\left\langle l_{5}, l_{7}, l_{8}\right\rangle$ & $\left\langle l_{8}, l_{7}, l_{6}\right\rangle$ \\
\hline$M_{3}$ & $\left\langle l_{6}, l_{5}, l_{7}\right\rangle$ & $\left\langle l_{8}, l_{2}, l_{3}\right\rangle$ & $\left\langle l_{7}, l_{4}, l_{2}\right\rangle$ & $\left\langle l_{2}, l_{4}, l_{7}\right\rangle$ \\
\hline$M_{4}$ & $\left\langle l_{7}, l_{8}, l_{5}\right\rangle$ & $\left\langle l_{7}, l_{8}, l_{2}\right\rangle$ & $\left\langle l_{3}, l_{6}, l_{1}\right\rangle$ & $\left\langle l_{1}, l_{5}, l_{2}\right\rangle$ \\
\hline
\end{tabular}

Step 2: Here, utilizing the PA operators on every individual decision matrices $D M^{k}(k=$ $1,2,3,4)$ and we obtain overall preferences values for the alternatives corresponding to the each decision-maker as follows

\begin{tabular}{llll}
\hline$C_{3 \times 1}^{1}$ & & $C_{3 \times 1}^{2}$ & \\
\hline$M_{1}$ & $\left\langle l_{3.673}, l_{6.6786}, l_{8.4837}\right\rangle$ & $M_{1}$ & $\left\langle l_{4.6147}, l_{3.5226}, l_{3.8862}\right\rangle$ \\
$M_{2}$ & $\left\langle l_{7.4683}, l_{5.4367}, l_{7.82}\right\rangle$ & $M_{2}$ & $\left\langle l_{6.1021}, l_{4.0812}, l_{7.2042}\right\rangle$ \\
$M_{3}$ & $\left\langle l_{6.6785}, l_{6.2815}, l_{7.6374}\right\rangle$ & $M_{3}$ & $\left\langle l_{5.1562}, l_{5.196}, l_{5.6749}\right\rangle$ \\
$M_{4}$ & $\left\langle l_{7.2851}, l_{4.9388}, l_{5.489}\right\rangle$ & $M_{3}$ & $\left\langle l_{5.6332}, l_{3.4215}, l_{5.2493}\right\rangle$ \\
\hline
\end{tabular}

Step 3: Here, we apply decision-maker's weight according to the relation $\sum_{k=1}^{r} \phi_{k} C_{s \times 1}^{k}$ and we obtain overall preferences values for the alternatives $M_{i}$ as follows

Step 4: Utilize the score function according to the Definition 3.2, we get, $S\left(\widetilde{L}_{11}\right)=7.5598$, $S\left(\widetilde{L}_{21}\right)=7.7282, S\left(\widetilde{L}_{31}\right)=7.6863, S\left(\widetilde{L}_{41}\right)=8.0594$. 


\begin{tabular}{llll}
\hline$C_{3 \times 1}^{3}$ & & $C_{3 \times 1}^{4}$ & \\
\hline$M_{1}$ & $\left\langle l_{4.3824}, l_{3.5729}, l_{5.0595}\right\rangle$ & $M_{1}$ & $\left\langle l_{6.3805}, l_{5.4693}, l_{6.0826}\right\rangle$ \\
$M_{2}$ & $\left\langle l_{7.3121}, l_{3.8494}, l_{4.9026}\right\rangle$ & $M_{2}$ & $\left\langle l_{5.1644}, l_{5.5765}, l_{7.1855}\right\rangle$ \\
$M_{3}$ & $\left\langle l_{6.0482}, l_{3.8743}, l_{6.263}\right\rangle$ & $M_{3}$ & $\left\langle l_{6.0949}, l_{4.1834}, l_{4.7231}\right\rangle$ \\
$M_{4}$ & $\left\langle l_{7.064}, l_{4.5249}, l_{6.8205}\right\rangle$ & $M_{4}$ & $\left\langle l_{5.144}, l_{7.1436}, l_{2.7626}\right\rangle$ \\
\hline
\end{tabular}

Overall evaluation of the alternatives

$\begin{array}{ll}M_{1} & \left\langle l_{4.8969}, l_{4.5906}, l_{5.6083}\right\rangle \\ M_{2} & \left\langle l_{6.702}, l_{4.6398}, l_{6.6492}\right\rangle \\ M_{3} & \left\langle l_{6.0368}, l_{4.8026}, l_{6.0118}\right\rangle \\ M_{4} & \left\langle l_{6.4541}, l_{4.771}, l_{4.9022}\right\rangle\end{array}$

Table 1 Score values of overall preferences values under different operators

\begin{tabular}{llllll}
\hline & $S\left(\widetilde{L}_{11}\right)$ & $S\left(\widetilde{L}_{21}\right)$ & $S\left(\widetilde{L}_{31}\right)$ & $S\left(\widetilde{L}_{41}\right)$ & Ranking order \\
\hline LGSFPA & 7.5598 & 7.7282 & 7.6863 & 8.0594 & $M_{4}>M_{2}>M_{3}>M_{1}$ \\
LGSFPWA & 7.5731 & 7.7482 & 7.7135 & 8.1232 & $M_{4}>M_{2}>M_{3}>M_{1}$ \\
LGSFPG & 7.0249 & 7.0328 & 7.0713 & 7.5033 & $M_{4}>M_{3}>M_{2}>M_{1}$ \\
LGSFPWG & 7.0014 & 7.0297 & 7.059 & 7.4668 & $M_{4}>M_{3}>M_{2}>M_{1}$ \\
\hline
\end{tabular}

Step 5: The ranking order of the score values is $S\left(\widetilde{L}_{41}\right)>S\left(\widetilde{L}_{21}\right)>S\left(\widetilde{L}_{31}\right)>S\left(\widetilde{L}_{11}\right)$. Therefore, $M_{4}$ is the best alternative.

On the other hand, if we utilize others aggregation operators namely LGSFPWA, LGSFPG and LGSFPWG, then we observe that $M_{4}$ is the best alternative. The ranking order under different proposed operators are shown in the Table 1.

\section{Sensitivity analysis and comparative analysis}

\subsection{Sensitivity analysis}

Sensitivity analysis determines how different values of an independent variable affect a particular dependent variable under a given set of assumptions. In other words, sensitivity analysis study show how various sources of uncertainty in a mathematical model contribute to the model's overall uncertainty. This technique is used within specific boundaries that depend on one or more input variables. Based on the numerical analysis results (case of PA operators related GSFN), we now calculate the corresponding outputs for changing input parameters one by one. Here, we make an attempt to compute the sensitivity analysis by changing the weights of the decision-makers within a certain range and observed the ranking order under different operators like LGSFPA, LGSFPWA, LGSFPG and LGSFPWG respectively as shown below in Table 2. From the Table 2, we observed that the most of the cases $M_{4}$ is the best alternative and $M_{1}$ is the worst alternative under the different operators. Also, Figs. 3, 4, 5 and 6 represent the associated weights of distinct ranking order of the alternatives under the different operators LGSFPA, LGSFPWA, LGSFPG and LGSFPWG respectively. 


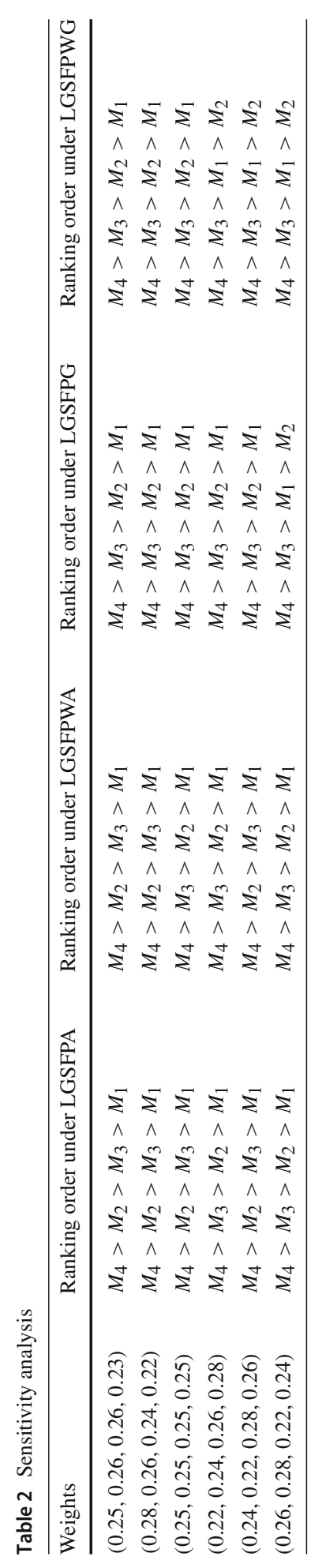




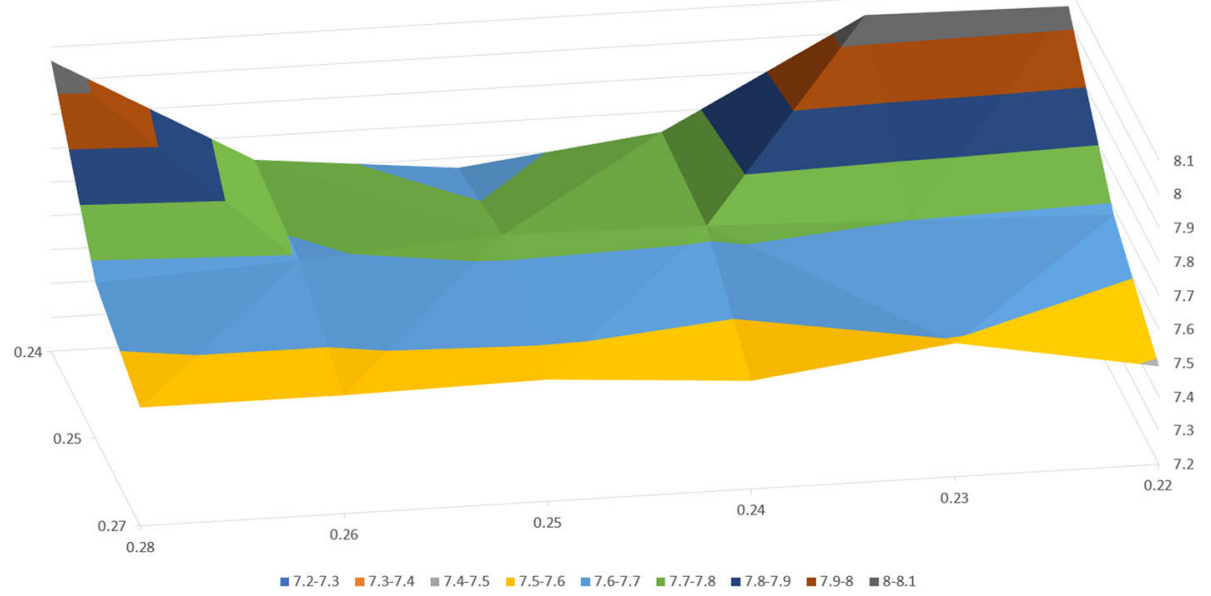

Fig. 3 The ranking order of the different alternatives under the operator LGSFPA

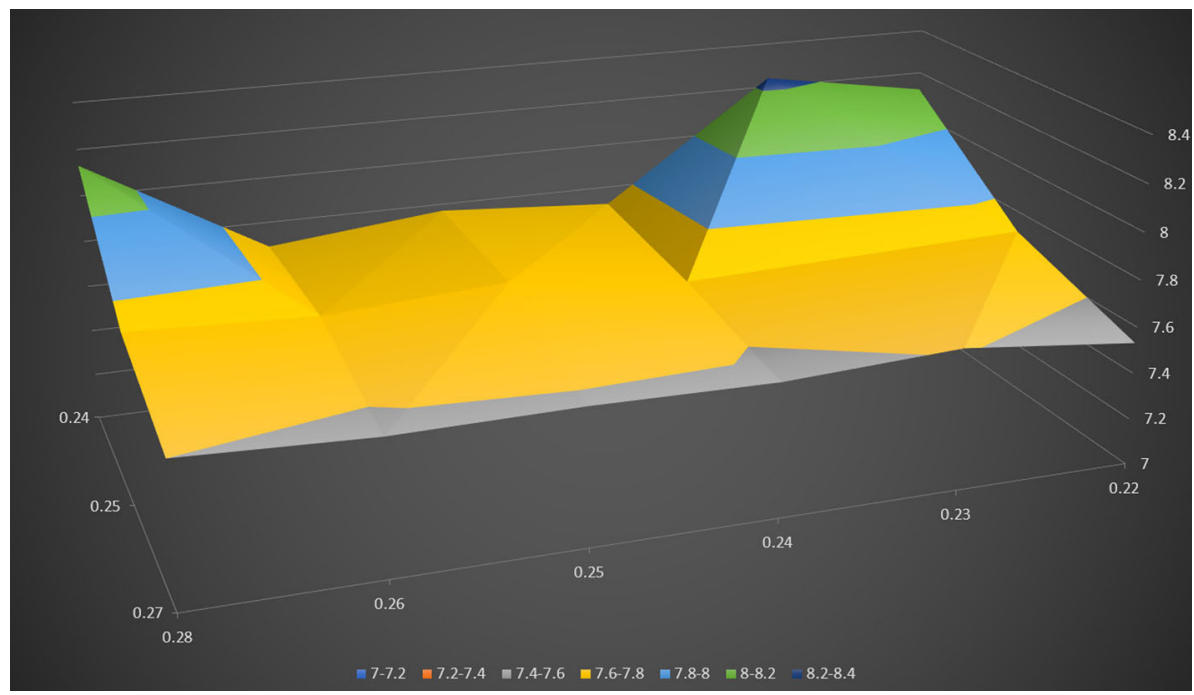

Fig. 4 The ranking order of the different alternatives under the operator LGSFPWA

\subsection{Comparative analysis}

Here to show the efficiency and superiority of our proposed technique, we have discussed a comparative study with the existing techniques which is shown in the following Table 3.

From Table 3, we observed that pre-existing PA operator IFEWPA (Jiang et al. 2018) cannot be applied in our set of data as the neutral part is the absence in the case of IFN. Also, we have seen that the aggregation operators by Garg (2018) cannot be used in our decision matrices as the neutral part is not present and the interrelation of the entities is not considered in this article. Moreover, the aggregation operators by Wei and Lu (2018) cannot apply here since the neutral part is absent as well as the information is not in a linguistic environment. Furthermore, the aggregation operators introduced by Jin et al. (2019) is not 


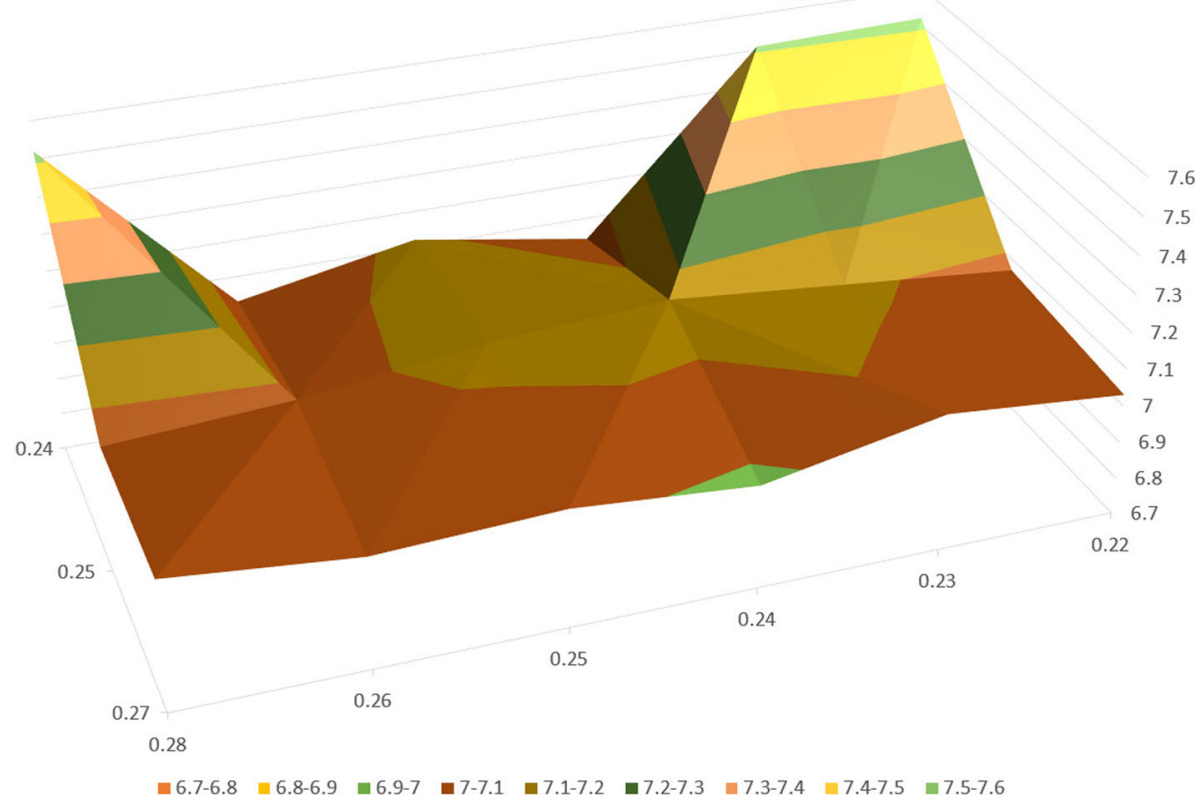

Fig. 5 The ranking order of the different alternatives under the operator LGSFPW

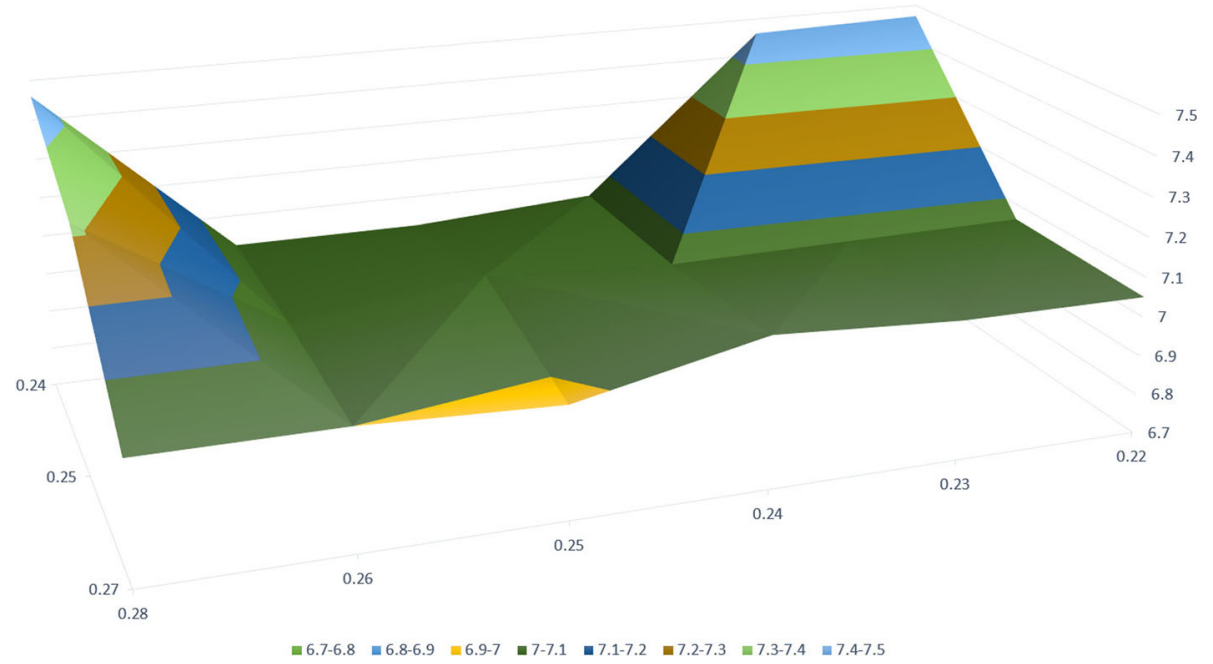

Fig. 6 The ranking order of the different alternatives under the operator LGSFPWG

applicable for determining ranking order as the interrelation of the entities is not considered in these aggregation operators and also if the data's like $\langle 0.9,0.8,0.7\rangle,\langle 0.9,0.6,0.8\rangle$ are present in the decision matrices cannot be capture by the spherical fuzzy environment.

From the above discussion, we can say that the pre-existing PA operators in a different environment like IFN, PYFN and SFN cannot handle the data given in the form of GSFNs. On the other hand, we observe that our proposed PA operators namely LGSFPA, LGSFPWA, 


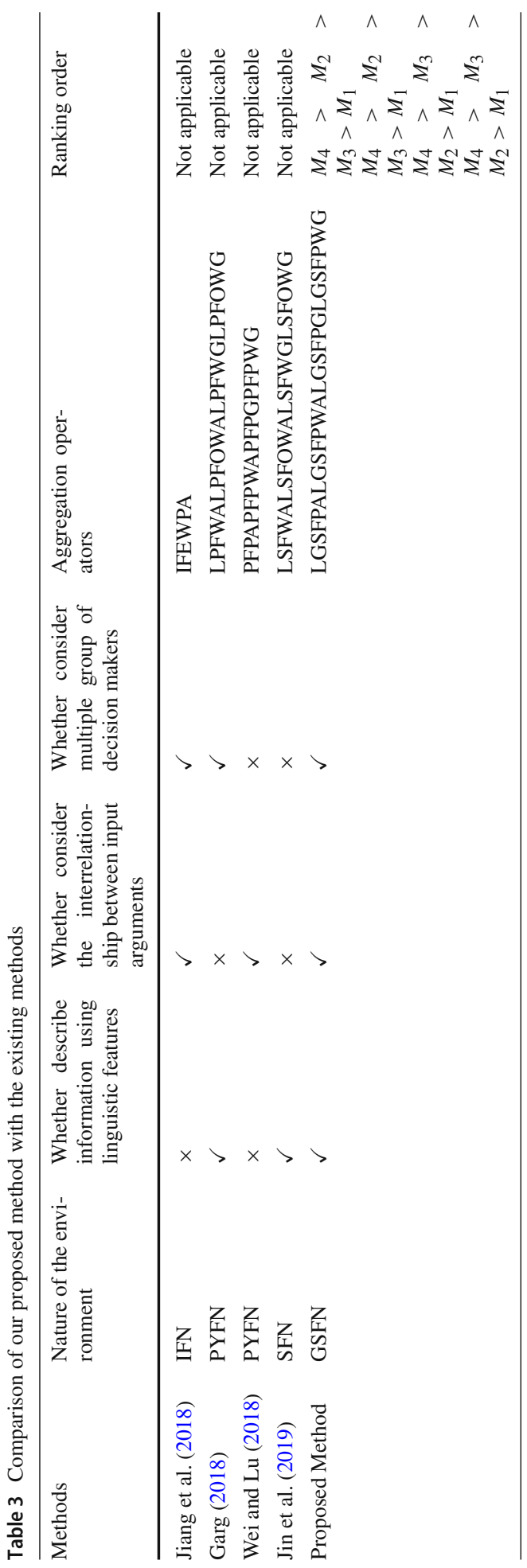


LGSFPG and LGSFPWG can solve the decision making problems, where the information is in the form of IFN, PYFN and SFN. This shows that our proposed method is much more efficient to deal with the above situation where aforesaid aggregation operators fail.

\section{Conclusion}

In this article, we have introduced a new linguistic generalized spherical fuzzy set by combining the idea of a generalized spherical fuzzy set and linguistic fuzzy set. Then, we discussed some basic algebraic properties of LGSFN. Here, new score and accuracy functions have been defined to compare any two LGSFNs. To utilize the benefit of GSFN and PA operators, we have defined various types of power aggregation operators namely LGSFPA, LGSFPWA, LGSFPG and LGSFPWG in the linguistic environment. Based on these proposed aggregation operators, we manifested an MCGDM technique in the LGSF environment. In this developed MCGDM technique, every alternative should have the same number of criteria because if alternatives have a different number of criteria, then we cannot apply the proposed aggregation operators to get the overall aggregation value of the alternatives under the different number of criteria. Finally, a numerical example was given to demonstrate the proposed technique. Also, sensitivity analysis was performed to show the reliability of the technique. Finally, a comparative analysis was given with existing methods to show the efficiency of the proposed technique.

In future work, the defined power aggregation operators for LGSFNs can be expanded to the other various type of uncertain and fuzzy environments to tackle the uncertainties in the decision-making technique. Further, researchers can immensely apply power aggregation operators for LGSFNs in numerous different research fields like image processing, engineering problem, mobile computing problems, big-data analysis, pattern recognition problems, diagnosis problems, realistic mathematical modeling, etc.

Acknowledgements In this article, the study of Tipu Sultan Haque is funded by Council of Scientific \& Industrial Research (CSIR), India (File no.-08/003(0136)/2019-EMR-I).

\section{Declarations}

Conflict of interest The authors have no conflict of interest in this research paper.

\section{References}

Ali Z, Mahmood T, Ullah K, Khan Q (2021) Einstein geometric aggregation operators using a novel complex interval-valued pythagorean fuzzy setting with application in green supplier chain management. Rep Mech Eng 2(1):105-134

Ashraf S, Abdullah S (2019) Spherical aggregation operators and their application in multi-attribute group decision making. Int J Intell Syst 34(3):493-523

Ashraf S, Abdullah S, Mahmood T (2018) GRA method based on spherical linguistic fuzzy Choquet integral environment and its application in multi-attribute decision-making problems. Math Sci 12:263-275. https://doi.org/10.1007/s40096-018-0266-0

Ashraf S, Abdullah S, Mahmood T, Ghani F, Mahmood T (2019) Spherical fuzzy sets and their applications in multi-attribute decision making problems. J Intell Fuzzy Syst 36(3):2829-2844. https://doi.org/10.3233/ JIFS- 172009

Attanassov KT (1986) Intuitionistic fuzzy sets. Fuzzy Sets Syst 20(1):87-96 
Chakraborty A, Mondal SP, Alam S, Mahata A (2020) Cylindrical neutrosophic single-valued number and its application in networking problem, multi-criterion group decision-making problem and graph theory. CAAI Trans Intell Technol 5(2):68-77

Chakraborty A, Mondal SP, Alam S, Dey A (2021) Classification of trapezoidal bipolar neutrosophic number, de-bipolarization technique and its execution in cloud service-based MCGDM problem. Complex Intell Syst 7:145-162. https://doi.org/10.1007/s40747-020-00170-3

Fang Z, Ye J (2017) Multiple attribute group decision-making method based on linguistic neutrosophic numbers. Symmetry 9:111

Garg H (2018) Linguistic Pythagorean fuzzy sets and its applications in multiattribute decision-making process. Int J Intell Syst 33(6):1234-1263

Garg H (2019) Novel neutrality aggregation operators-based multiattribute group decision making method for single-valued neutrosophic numbers. Soft Comput. https://doi.org/10.1007/s00500-019-04535-w

Garg H (2021) T-spherical fuzzy power aggregation operators and their applications in multi-attribute decision making. J Ambient Intell Humaniz Comput. https://doi.org/10.1007/s12652-020-02600-z

Garg H, Nancy (2018) Linguistic single-valued neutrosophic prioritized aggregation operators and their applications to multiple-attribute group decision-making. J Ambient Intell Humaniz Comput 9(6):1975-1997

Gündogdua FK, Kahramana C (2019) Spherical fuzzy sets and spherical fuzzy TOPSIS method. J Intell Fuzzy Syst 36(1):337-352. https://doi.org/10.3233/JIFS-181401

Güner E, Aygün H (2020) Generalized spherical fuzzy Einstein aggregation operators: application to multicriteria group decision-making problems. Conf Proc Sci Technol 3(2):227-235

Haque TS, Chakraborty A, Mondal SP, Alam S (2021) A New Exponential Operational Law For Trapezoidal Neutrosophic Number and Pollution in Megacities related MCGDM Problem, Journal of Ambient Intelligence and Humanized Computing

Haque TS, Chakraborty A, Mondal SP, Alam S (2020) A new approach to solve multi-criteria group decision making problems by exponential operational law in generalised spherical fuzzy environment. CAAI Trans Intell Technol 5(2):106-114. https://doi.org/10.1049/trit.2019.0078

Jiang W, Wei B, Liu X, Li X, Zheng H (2018) Intuitionistic fuzzy power aggregation operator based on entropy and its application in decision making. Int J Intell Syst 33(1):49-67

Jin H, Ashraf S, Abdullah S, Qiyas M, Bano M, Zeng S (2019) Linguistic spherical fuzzy aggregation operators and their applications in multi-attribute decision making problems. Mathematics 7:413-435. https://doi. org/10.3390/math7050413

Jin H, Ashraf S, Abdullah S, Qiyas M, Bano M, Zeng S (2019) Linguistic spherical fuzzy aggregation operators and their applications in multi-attribute decision making problems. Mathematics 7:413. https://doi.org/ $10.3390 /$ math7050413

Jin Y, Kousar Z, Ullah K, Mahmood T (2021) Approach to multi-attribute decision-making methods for performance evaluation process using interval-valued T-spherical fuzzy Hamacher aggregation information. Axioms 10(3):145. https://doi.org/10.3390/axioms10030145

Karaaslan F (2018) Multi-criteria decision making method based on similarity measures under single-valued neutrosophic refined and interval neutrosophic refined environments. Int J Intell Syst 33(5):928-952

Karaaslan F, Dawood MAD (2021) Complex T-spherical fuzzy Dombi aggregation operators and their applications in multiple-criteria decision-making. Complex Intell Syst. https://doi.org/10.1007/s40747-02100446-2

Karaaslan F, Hayat K (2018) Some new operations on single-valued neutrosophic matrices and their applications in multi-criteria group decision making. Appl Intell 48(2):4594-4614

Li YY, Zhang HY, Wang JQ (2017) Linguistic neutrosophic sets and its application to multi-criteria decisionmaking problems. Int J Uncertain Quantif 7(2):135-154

Liu P, Zhu B, Wang P, Shen M (2020) An approach based on linguistic spherical fuzzy sets for public evaluation of shared bicycles in China. Eng Appl Artif Intell 87:1-15

Mahmood T, Ullah K, Khan Q, Jan N (2019) An approach toward decision-making and medical diagnosis problems using the concept of spherical fuzzy sets. Neural Comput Appl 31:7041-7053. https://doi.org/ 10.1007/s00521-018-3521-2

Munir M, Kalsoom H, Ullah K, Mahmood T, Chu Y-M (2020) T-spherical fuzzy einstein hybrid aggregation operators and their applications in multi-attribute decision making problems. Symmetry 12:365. https:// doi.org/10.3390/sym12030365

Nancy HG (2019) Linguistic single-valued neutrosophic power aggregation operators and their applications to group decision-making problems. IEEE/CAA J Autom Sin. https://doi.org/10.1109/JAS.2019.1911522

Pamučar D, Janković A (2020) The application of the hybrid interval rough weighted Power-Heronian operator in multi-criteria decision making. Oper Res Eng Sci Theory Appl 3(2):54-73 
Rahman K (2019) A series of generalized induced Einstein aggregation operators and their application to group decision-making process based on Pythagorean fuzzy numbers. Granul Comput. https://doi.org/ 10.1007/s41066-019-00184-4

Sinani F, Erceg Z, Vasiljević M (2020) An evaluation of a third-party logistics provider: the application of the rough Dombi-Hamy mean operator. Decis Mak Appl Manag Eng 3(1):92-107

Smarandache F (1999) A unifying field in logics. Neutrosophy: neutrosophic probability, set and logic. American Research Press, Rehoboth

Smarandache F (2017) Neutrosophic perspectives: triplets, duplets, multisets, hybrid operators, modal logic, Hedge algebras. And applications, Second extended and improved edition. Pons Publishing House, Brussels, pp 346-374

Smarandache F (2019) Neutrosophic set is a generalization of intuitionistic fuzzy set, inconsistent intuitionistic fuzzy set (picture fuzzy set, ternary fuzzy set), pythagorean fuzzy set, spherical fuzzy set, and q-rung orthopair fuzzy set, while neutrosophication is a generalization of regret theory, grey system theory, and three-ways decision (revisited). J New Theory 29:1-31

Ullah K, Garg H, Mahmood T, Jan T, Ali Z (2020) Correlation coefficients for T-spherical fuzzy sets and their applications in clustering and multi-attribute decision making. Soft Comput 24:1647-1659. https://doi. org/10.1007/s00500-019-03993-6

Uluçay V, Deli I, Sahin M (2019) Intuitionistic trapezoidal fuzzy multi-numbers and its application to multicriteria decision-making problems. Complex Intell Syst 5:65-78. https://doi.org/10.1007/s40747-0180074-z

Wei G, Lu M (2018) Pythagorean fuzzy power aggregation operators in multiple attribute decision making. Int J Intell Syst 33:169-186

$\mathrm{Xu} \mathrm{Z}$ (2011) Approaches to multiple attribute group decision making based on intuitionistic fuzzy power aggregation operators. Knowl Based Syst 24:749-760

Xu ZS, Yager RR (2010) Power-geometric operators and their use in group decision making. IEEE Trans Fuzzy Syst 18(1):94-105

Yager RR (2013) Pythagorean fuzzy subsets. In: Proceedings joint IFSA world congress and NAFIPS annual meeting, Edmonton, Canada, pp 57-61

Yager RR (2001) The power average operator. IEEE Trans Syst Man Cybern A 31:724-731

Yager RR, Abbasov AM (2013) Pythagorean membeship grades, complex numbers and decision making. Int J Intell Syst 28:436-452

Yang L, Li B (2016) A multi-criteria decision-making method using power aggregation operators for singlevalued neutrosophic sets. Int J Database Theory Appl 9(2):23-32

Zadeh LA (1965) Fuzzy sets. Inf Control 8(3):338-353

Zadeh LA (1975) The concept of a linguistic variable and its application to approximate reasoning: part-1. Inf Sci 8:199-251

Publisher's Note Springer Nature remains neutral with regard to jurisdictional claims in published maps and institutional affiliations. 